\title{
ON $N$-PARAMETER FAMILIES AND INTERPOLATION PROBLEMS FOR NONLINEAR ORDINARY DIFFERENTIAL EQUATIONS( ${ }^{1}$ )
}

\author{
BY \\ PHILIP HARTMAN
}

\begin{abstract}
Let $y=\left(y_{0}, \ldots, y_{N-1}\right)$. This paper is concerned with the existence of solutions of a system of ordinary differential equations $y^{\prime}=g(t, y)$ satisfying interpolation conditions $\left({ }^{*}\right) y_{0}\left(t_{j}\right)=c_{j}$ for $j=1, \ldots, N$ and $t_{1}<\cdots<t_{N}$. It is shown that, under suitable conditions, the assumption of uniqueness for all such problems and of "local" solvability (i.e., for $t_{1}, \ldots, t_{N}$ on small intervals) implies the existence for arbitrary $t_{1}, \ldots, t_{N}$ and $c_{1}, \ldots, c_{N}$. A result of Lasota and Opial shows that, in the case of a second order equation for $y_{0}$, the assumption of uniqueness suffices, but it will remain undecided if the assumption of "local" solvability can be omitted in general. More general interpolation conditions involving $N$ points, allowing coincidences, are also considered.

Part I contains the statement of the principal results for interpolation problems and those proofs depending on the theory of differential equations. Actually, the main theorems are consequences of results in Part II dealing with " $N$-parameter families" and " $N$-parameter families with pseudoderivatives." A useful lemma states that if $F$ is a family of continuous functions $\left\{y^{0}(t)\right\}$ on an open interval $(a, b)$, then $F$ is an $N$-parameter family (i.e., contains a unique solution of the interpolation conditions $\left(^{*}\right)$ for arbitrary $t_{1}<\cdots<t_{N}$ on $(a, b)$ and $\left.c_{1}, \ldots, c_{N}\right)$ if and only if (i) $y^{0}, z^{0} \in F$ implies $y^{0}-z^{0} \equiv 0$ or $y^{0}-z^{0}$ has at most $N$ zeros; (ii) the set $\Omega$ $\equiv\left\{\left(t_{1}, \ldots, t_{N}, y^{0}\left(t_{1}\right), \ldots, y^{0}\left(t_{N}\right)\right): a<t_{1}<\cdots<t_{N}<b\right.$ and $\left.y^{0} \in F\right\}$ is open in $R^{2 N}$; (iii) $y^{1}, y^{2}, \ldots, \in F$ and the inequalities $y^{n}(t) \leqq y^{n+1}(t)$ for $n=1,2, \ldots$ or $y^{n}(t) \geqq y^{n+1}(t)$ for $n=1,2, \ldots$ on an interval $[\alpha, \beta] \subset(a, b)$ imply that either $y^{0}(t)=\lim y^{n}(t)$ exists on $(a, b)$ and $y^{0} \in F$ or $\lim \left|y^{n}(t)\right|=\infty$ on a dense set of $(a, b)$; and finally, (iv) the set $S(t)=\left\{y^{0}(t): y^{0} \in F\right\}$ is not bounded from above or below for $a<t<b$. The notion of pseudoderivatives permits generalizations to interpolation problems involving some coincident points.
\end{abstract}

\section{PART I. INTERPOLATION PROBLEMS}

1. Main results. All variables and functions below are real valued. Consider an $N$ th order differential equation, which we write as a first order system for the vector $y=\left(y_{0}, \ldots, y_{N-1}\right)$,

$$
y_{0}^{\prime}=y_{1}, \ldots, y_{N-2}^{\prime}=y_{N-1}, \quad y_{N-1}^{\prime}=Y\left(t, y_{0}, \ldots, y_{N-1}\right) .
$$

Received by the editors February 26, 1970.

AMS 1969 subject classifications. Primary 3436, 4110.

Key words and phrases. Ordinary nonlinear differential equations, multiple point conditions, interpolation, $N$-parameter family, pseudoderivatives.

(1) Research partially sponsored by the Air Force Office of Scientific Research, Office of Aerospace Research, United States Air Force, under AFOSR Contract No. F44620-67-C-0098. This paper also represents results obtained at the Courant Institute of Mathematical Sciences of New York University, under the sponsorship of the Sloan Foundation. 
The questions to be discussed in this paper include, for example, the problem of the existence of a solution of (1.1) satisfying boundary conditions at $N$ distinct points,

$$
y_{0}\left(t_{j}\right)=c_{j} \text { for } j=1, \ldots, N \text {, where } t_{1}<\cdots<t_{N},
$$

or boundary conditions at $N$ points, allowing coincidences,

$$
\begin{aligned}
y_{k}\left(t_{j}\right)=c_{j k} \text { for } j & =1, \ldots, m \text { and } k=0, \ldots, n(j)-1, \\
& t_{1}<\cdots<t_{m} ; n(j) \geqq 1 ; n(1)+\cdots+n(m)=N .
\end{aligned}
$$

We shall also be concerned with the situation where (1.1) is replaced by a more general first order system

$$
y^{\prime}=g(t, y)
$$

$y=\left(y_{0}, \ldots, y_{N-1}\right)$ and $g=\left(g_{0}, \ldots, g_{N-1}\right)$. In the case $N=2$, we have the following result:

THEOREM I 1.1. Let $J=(a, b],-\infty \leqq a<b<\infty$, be a half-open interval and $J^{0}$ $=(a, b)$ its interior. Let $N=2, y=\left(y_{0}, y_{1}\right)$, and $g=\left(g_{0}, g_{1}\right)$. Suppose that $g(t, y) \in C^{0}\left(J \times R^{2}, R^{2}\right)$ is such that $\left(\mathrm{i}_{0}\right) g_{0}\left(t, y_{0}, y_{1}\right)$ is an increasing function of $y_{1}$ for fixed $\left(t, y_{0}\right), t \in J^{0}$ satisfying $g_{0} \rightarrow \pm \infty$ as $y_{1} \rightarrow \pm \infty$ uniformly on $\left(t, y_{0}\right)-$ compacts; (ii) all solutions of (1.4) exist on $J$; and (iii $\left.{ }_{0}\right)$ if $y=\left(y_{0}(t), y_{1}(t)\right)$ and $z=\left(z_{0}(t), z_{1}(t)\right)$ are distinct solutions of (1.4), then $y_{0}(t)-z_{0}(t)$ has at most one zero on $J^{0}$ (that is, (1.4) and (1.2), with $N=2$, has at most one solution if $t_{1}, t_{2} \in J^{0}$ for all $\left.c_{1}, c_{2}\right)$. Then every boundary value problem (1.4), (1.2) has at least one solution.

This generalizes slightly the result of Lasota and Opial [10] in replacing their second order equation (1.1), with $N=2$, by a more general first order system (1.4), with $N=2$. It also (1) replaces their open interval by a half-open interval and (2) omits their assumption of the uniqueness for initial value problems. As to (1), (2), and other generalizations for second order problems (1.1) and (1.2), with $N=2$, see Schrader and Waltman [17] and references there to Jackson, Schrader, Waltman, Bailey, and Shampine. Devices of [10], [17] can be used to obtain corresponding generalizations of Theorem I 1.1.

THEOREM I 1.2. Let $J, y, g$ be as in Theorem I 1.1 except that condition (iii ${ }_{0}$ ) is replaced by the following: (iii*) there is at most one solution of (1.4) satisfying

$$
y_{0}\left(t_{1}\right)=c_{1} \text { and } y_{1}\left(t_{2}\right)=c_{2}, \quad t_{1} \neq t_{2},
$$

for arbitrary $c_{1}, c_{2}$ and $t_{1}, t_{2} \in J^{0}$. Then condition (iii ${ }_{0}$ ), hence the conclusion of Theorem I 1.1, holds; also, every boundary value problem (1.4), (1.5) has at least one solution if $t_{1} \in J^{0}, t_{2} \in J$.

Theorem I 1.2 contains the result of Lasota and Luczyński [8] for a second order equation (1.1), $N=2$, with (1.5) replaced by $y_{0}\left(t_{1}\right)=c_{1}$ and $y_{0}^{\prime}\left(t_{2}\right)+\gamma y_{0}\left(t_{2}\right)=c_{2}$ 
where $t_{1} \neq t_{2}$, and $\gamma$ is a fixed constant. In order to see this, it suffices to replace the equation $y_{0}^{\prime \prime}=Y\left(t, y_{0}, y_{0}^{\prime}\right)$ by a first order system for $y=\left(y_{0}, y_{1}\right)=\left(y_{0}, y_{0}^{\prime}+\gamma y_{0}\right)$,

$$
y_{0}^{\prime}=y_{1}-\gamma y_{0}, \quad y_{1}^{\prime}=Y\left(t, y_{0}, y_{1}-\gamma y_{0}\right)+\gamma\left(y_{1}-\gamma y_{0}\right) .
$$

We cannot decide if the analogue of Theorem I 1.1 holds for $N>2$; such an analogue becomes correct for open intervals if a condition of "local solvability" is added.

[Added in proof (October 26, 1970). After the completion of this paper, Professor P. Waltman showed me a preprint of the forthcoming paper Existence and uniqueness of solutions of boundary value problems for third order differential equations by L. Jackson and K. Schrader. These authors obtain an analogue for $N=3$ of the Lasota-Opial [10] result for $N=2$; i.e., if $N=3$ and (1.4) is replaced by (1.1), then the condition ( iii $_{0}$ ) of "local solvability" can be omitted in the following theorem.]

THEOREM I 1.3. Let $N>2$ and $J^{0}=(a, b)$ an open interval; also $y=\left(y_{0}, \ldots, y_{N-1}\right)$ and $g=\left(g_{0}, \ldots, g_{N-1}\right)$. Let $g(t, y) \in C^{0}\left(J^{0} \times R^{N}, R^{N}\right)$ be such that $\left(\mathrm{i}_{0}\right)$ all solutions of (1.4) exist on $J^{0}$; $\left(\mathrm{ii}_{0}\right)$ if $y(t), z(t)$ are distinct solutions of $(1.4)$, then $y_{0}(t)-z_{0}(t)$ has at most $N-1$ zeros on $J^{0}\left(\right.$ that is, (1.4) and (1.2) has at most one solution if $t_{1}, \ldots, t_{N} \in J^{0}$ for all $\left.c_{1}, \ldots, c_{N}\right)$; and $\left(\mathrm{iii}_{0}\right)$ the boundary value problems (1.4), (1.2) are locally solvable at every point $t_{0} \in J^{0}$, that is, for every $t_{0} \in J^{0}$, there exists an open interval $J\left(t_{0}\right), t_{0} \in J\left(t_{0}\right) \subset J^{0}$ such that (1.4), (1.2) has a solution for all distinct $t_{1}, \ldots, t_{N} \in J\left(t_{0}\right)$ and all $c_{1}, \ldots, c_{N}$. Then every boundary value problem (1.4), (1.2) has a unique solution.

We can obtain a theorem for half-open intervals by adding an additional condition ( $\left.i^{*}\right)$. (Incidentally, this condition always holds if $N=2$, under the assumptions of Theorem I 1.1.)

Corollary I 1.1. Let $N>2, J=(a, b]$ and $J^{0}=(a, b)$. Let $g(t, y) \in C^{0}\left(J \times R^{N}, R^{N}\right)$ be such that (i) all solutions of (1.4) exist on $J$; (i*) if $y_{n}=\left(y_{n 0}(t), \ldots, y_{n, N-1}(t)\right)$ is a solution of (1.4) for $n=0,1, \ldots$, and $y_{n 0}(t) \rightarrow y_{00}(t)$ uniformly on compacts of $J^{0}$, as $n \rightarrow \infty$, then $y_{n 0}(b) \rightarrow y_{00}(b)$; conditions $\left(\mathrm{ii}_{0}\right)$, ( $\left(\mathrm{ii}_{0}\right)$ of Theorem $\mathrm{I} 1.3$ hold on $J^{0}$. Then every boundary value problem (1.4), (1.2), with $t_{j} \in J$, has at least one solution.

We can deal with the more general boundary conditions (1.3) under additional hypotheses.

THEOREM I $1.4(\alpha)$. Let $N>2, J^{0}, g$ be as in Theorem I 1.3 and satisfy the following additional hypothesis: $\left(\mathrm{iv}_{K}\right)$ let $1 \leqq K \leqq N$ and if $y=\left(y_{0}(t), \ldots, y_{N-1}(t)\right)$ is a solution of (1.4), then $y_{1}(t), \ldots, y_{K-1}(t)$ are pseudoderivatives of $y_{0}$ on $J^{0}$ in the sense of Definition 1.3 in $\$ 1$ of Part II below; cf., e.g., Propositions 2.1 and 2.2 in Part II below. Then every boundary value problem (1.4), (1.3), with $1 \leqq n(j) \leqq K$ and $n(1)+\cdots+n(m)=N$, has at least one solution. $(\beta)$ Furthermore, if $K=N$ and $\left(\mathrm{v}_{0}\right)$ 
solutions of (1.4) are uniquely determined by initial conditions at points $t_{0} \in J^{0}$, then a solution of (1.4), (1.3) is unique.

A corresponding result on a half-open interval is the following:

Corollary I 1.2. Let $N>2, \quad 1 \leqq K \leqq N, \quad J=(a, b], \quad J^{0}=(a, b), \quad$ and $g \in$ $C^{0}\left(J \times R^{N}, R^{N}\right)$. Assume conditions (i), ( $\left.\mathrm{i}^{*}\right)$ of Corollary $\mathrm{I} 1.1$; also conditions $\left(\mathrm{ii}_{0}\right)$, (iii $\left.{ }_{0}\right)$ of Theorem I 1.3 and condition $\left(\mathrm{iv}_{K}\right)$ of Theorem I 1.4( $\alpha$ ) on $J^{0}$. Then every boundary value problem (1.4), (1.3), with $1 \leqq n(j) \leqq K$ and $n(1)+\cdots+n(m)=N$, has a solution, provided that $t_{m}<b$ or that $t_{m}=b, n(m)=1$.

Note that when (1.4) reduces to (1.1), that is $g_{k}=y_{k+1}$ for $k=0, \ldots, N-2$ and $g_{N-1}=Y$, then the condition ( iv $_{N}$ ) of Theorem I $1.4(\alpha)$ is trivially satisfied.

Also, in the case (1.1) of (1.4), Theorem 8 of Lasota and Opial [9] implies that the condition ( $\mathrm{iii}_{0}$ ) of Theorem I 1.3 of "local solvability" at $t=t_{0} \in J^{0}$ is satisfied if there exists a continuous function $M(t)$ such that

$$
\left|Y\left(t, y_{0}, \ldots, y_{N-1}\right)\right| \leqq M(t)\left(1+\sum_{k=0}^{N-1}\left|y_{k}\right|\right)
$$

for small $\left|t-t_{0}\right|$ and all $y=\left(y_{0}, \ldots, y_{N-1}\right)$; cf. also Lemma I 1.1 below. Of course, if there exists a continuous $M(t) \in C^{0}\left(J^{0}\right)$ satisfying (1.6), then conditions ( $\left.\mathrm{i}_{0}\right)$, (iii $)$ of Theorem I 1.3 hold. When (1.4) is "nearly" an $N$ th order equation, one can obtain an analogue of the sufficient condition (1.6) for "local solvability."

LEMMA I 1.1. Let $N \geqq 2$, and the first $N-1$ components of $g=\left(g_{0}, \ldots, g_{N-1}\right) \in$ $C^{0}\left(J \times R^{N}, R^{N}\right)$ have the form

$$
g_{k}(t, y)=\sum_{j=0}^{k+1} P_{k j}(t, y) y_{j} \quad \text { for } k=0, \ldots, N-2,
$$

where $P_{k j}(t, y) \in C^{0}\left(J \times R^{N}, R\right)$, and there exist constants $M, c>0$ such that

$$
\begin{gathered}
\left|P_{k j}(t, y)\right| \leqq M \text { for } j=0, \ldots, k+1 \text { and } k=0, \ldots, N-2, \\
P_{k, k+1}(t, y) \geqq c>0 \quad \text { for } k=0, \ldots, N-2 .
\end{gathered}
$$

In addition, suppose that the Nth component $g_{N-1}$ of $g$ satisfies

$$
\left|g_{N-1}(t, y)\right| \leqq M\left(1+\sum_{k=0}^{N-1}\left|y_{k}\right|\right) .
$$

Then there exists a constant $\delta>0$, depending only on $N$ and $M$, such that if the length of $J$ satisfies $b-a<\delta$, then every boundary value problem (1.4), (1.3) has at least one solution.

It will be clear from the proof that, with the same $\delta$, other classes of boundary value problems have solutions; for example, (1.4) and

$$
y_{k}\left(t_{k}\right)=c_{k} \text { for } k=1, \ldots, N \text { and } t_{k} \gtreqless t_{j} .
$$


Compare this result with [16] and [11], where other problems are treated, but the $\delta$ there depends on a fixed set of boundary conditions (instead of allowing all conditions (1.3) [or (1.11)] as in Lemma I 1.1).

The condition of "local solvability" ( iii $_{0}$ ) in Theorem I 1.4 and its corollary can be weakened to a type of "compactness" assumption $\left(\mathrm{vi}_{0}\right)$, suggested by the Beckenbach-Tornheim continuity theorem for $N$-parameter families (see Lemma I 1.1 in Part II below).

TheOREM I 1.5. Let $N>2, J^{0}=(a, b)$ an open interval, $y=\left(y_{0}, \ldots, y_{N-1}\right)$ and $g=\left(g_{0}, \ldots, g_{N-1}\right)$. Let $g(t, y) \in C^{0}\left(J^{0} \times R^{N}\right)$ be such that conditions $\left(\mathrm{i}_{0}\right)$, (ii $\left.{ }_{0}\right)$ of Theorem I 1.3 and conditions $\left(\mathrm{iv}_{N}\right),\left(\mathrm{v}_{0}\right)$ of Theorem $\mathrm{I} 1.4$ hold. In addition, suppose that $\left(\mathrm{vi}_{0}\right)$ if $y_{n}(t)=\left(y_{n 0}(t), \ldots, y_{n, N-1}(t)\right)$ for $n=1,2, \ldots$ is any sequence of solutions of (1.4) satisfying

$$
y_{n 0} \leqq y_{n+1,0} \quad \text { for } n=1,2, \ldots \text { or } \quad y_{n 0} \geqq y_{n+1,0} \quad \text { for } n=1,2, \ldots,
$$

on some compact $[\alpha, \beta] \subset J^{0}$, then either

$$
\begin{aligned}
& y_{0}(t)=\lim _{n \rightarrow \infty} y_{n 0}(t) \text { exists on } J^{0} \text { and there } \\
& \text { is a solution }\left(y_{0}(t), \ldots, y_{N-1}(t)\right) \text { of }(1.4)
\end{aligned}
$$

or

$$
\lim _{n \rightarrow \infty}\left|y_{n 0}(t)\right|=\infty \text { on a dense set of } J^{0} .
$$

Then every boundary value problem (1.4), (1.3) has a unique solution.

It can be mentioned that if (1.4) reduces to an $N$ th order equation (1.1) and if, for every small interval $[\alpha, \beta] \subset J^{0}$, there exists a constant $M=M(\alpha, \beta)$ such that $g_{N-1}=Y$ satisfies $(1.10)$ for $t \in[\alpha, \beta]$, then condition $\left(v_{0}\right)$ holds even if (1.14) is replaced by

$$
\lim _{n \rightarrow \infty}\left|y_{n 0}(t)\right|=\infty \quad \text { except at } N-1 \text { points of } J^{0}
$$

cf., e.g., the proof of Lemma 7.1 in [5, p. 479].

Theorem I 1.1 will be proved in 2 , Theorem I 1.2 in 33 , and Lemma I 1.1 in $\S 4$. The remaining results do not really depend on the theory of ordinary differential equations and are consequences of results on " $N$-parameter families" or on " $N$-parameter families with pseudoderivatives" to be discussed in Part II below. Thus Theorem I 1.3 is a corollary of Theorem II 1.1 below, while Corollary I 1.1 follows from Corollary II 1.1, Theorem I 1.4 from Theorem II 1.2, Corollary I 1.2 from Corollary II 1.2, and Theorem I 1.5 from Theorem II 1.3.

For older references to Nicolleti, Conti, et al., see the bibliographies of [3], [16].

2. Proof of Theorem I 1.1. In proving the existence of a solution of (1.4), (1.2) with $N=2$, there is no loss of generality in supposing that $t_{2}=b$. Let $t_{1}, c_{1}$ be given, 
$a<t_{1}<b$. It suffices to show that if $S$ is the set of $y_{0}(b)$-values, where $y(t)$ $=\left(y_{0}(t), y_{1}(t)\right)$ is a solution of (1.4) satisfying $y_{0}\left(t_{1}\right)=c_{1}$, then $S=(-\infty, \infty)$.

Let $K>0$ and $S_{K 1}$ the set of $y(b)$-values of solutions of (1.4) satisfying the initial conditions

$$
y\left(t_{1}\right)=\left(c_{1}, d\right) \text { for }|d| \leqq K .
$$

Then $S_{K 1}$ is a closed connected set in the $y$-plane; Fukuhara [4]. Thus if $S_{K}$ is the projection of $S_{K 1}$ on the $y_{0}$-axis, i.e., if $S_{K}$ is the set of $y_{0}(b)$-values of solution of (1.4) satisfying (2.1), then $S_{K}$ is a $y_{1}$-interval (possibly a point) which is nondecreasing with $K$, and $S$ is the union of $S_{K}, K>0$. Hence $S$ is connected.

Suppose that $S \neq(-\infty, \infty)$, e.g., that $S$ is bounded from above, say $S \subset(-\infty, M)$. Let $y=y_{(n)}(t)$ be a sequence of solutions of (1.4), where

$$
y_{(n)}\left(t_{1}\right)=\left(c_{1}, n\right) \text { for } n=1,2, \ldots
$$

If $t^{1}, t^{2}, \ldots$ is a sequence of $t$-values on a compact subinterval of $J^{0}$, then

$$
\left\|y_{(n)}\left(t^{n}\right)\right\| \rightarrow \infty \quad \text { as } n \rightarrow \infty ;
$$

for otherwise, the sequence $\left\{y_{(n)}(t)\right\}$ has a subsequence which tends to a solution of (1.4) uniformly on compacts of $J$; cf. [6, pp. 14-15]. By condition (iii ${ }_{0}$ ), the first component $y_{n 0}(t)$ of $y_{(n)}(t)$ satisfies

$$
y_{n 0}(s)>y_{n+1,0}(s) \text { and } y_{n 0}(t)<y_{n+1,0}(t) \text { for } a<s<t_{1}<t<b .
$$

The sequence $\left\{y_{n 0}(t)\right\}$ cannot be uniformly bounded on any subinterval of $J^{0}$. For suppose that there is a constant $C$ such that $\left|y_{n 0}(t)\right| \leqq C$ on $[\alpha, \beta] \subset J^{0}$. Then there exists a $t^{n} \in(\alpha, \beta)$, where $\left|y_{n 0}^{\prime}\left(t^{n}\right)\right| \leqq 2 C /(\beta-\alpha)$. Condition $\left(\mathrm{i}_{0}\right)$ then implies that $\left\|y_{(n)}\left(t^{n}\right)\right\|$ is bounded. But this contradicts (2.3). Hence there exist sequences $\left\{s_{n}\right\},\left\{t_{n}\right\}$ such that

$$
\begin{gathered}
t_{1}>s_{n} \rightarrow t_{1} \text { and } t_{1}<t_{n} \rightarrow t_{1}, \\
y_{n 0}\left(s_{n}\right) \rightarrow-\infty \text { and } y_{n 0}\left(t_{n}\right) \rightarrow \infty \text { as } n \rightarrow \infty .
\end{gathered}
$$

Let $y=y^{*}(t)$ be any solution of (1.4) satisfying $y_{0}^{*}(b)=M$, so that $S \subset(-\infty, M)$ implies that $y_{0}^{*}(b)>y_{n 0}(b)$ for all $n$. But, by $(2.5), y_{0}^{*}\left(t_{n}\right)<y_{n 0}\left(t_{n}\right), y_{0}^{*}\left(s_{n}\right)>y_{n 0}\left(s_{n}\right)$ for large $n$. Hence, $y_{0}^{*}-y_{n 0}$ has two zeros on $(a, b)$. This contradicts (iii ${ }_{0}$ ) and shows that $S$ is not bounded from above. Similarly, it is not bounded from below. This completes the proof of Theorem I 1.1.

3. Proof of Theorem I 1.2. We first verify that the conditions of Theorem I 1.2 imply ( (iii $_{0}$ ) in Theorem I 1.1. Let $y(t), z(t)$ be distinct solutions of (1.4) satisfying $y_{0}=z_{0}$ for some point $t=t_{1} \in J^{0}$. It will be shown that $y_{0} \neq z_{0}$ for $t_{1} \neq t \in J^{0}$. Suppose, for example that $y_{1}(t)<z_{1}(t)$ for $t_{1}<t<b$. In particular, $y_{1}\left(t_{1}\right) \leqq z_{1}\left(t_{1}\right)$. On the one hand, if $y_{1}\left(t_{1}\right)=z_{1}\left(t_{1}\right)$, then, by (iii*), $y_{0}(t) \neq z_{0}(t)$ for $t_{1} \neq t \in J^{0}$. On the other hand, if $y_{1}\left(t_{1}\right)<z_{1}\left(t_{1}\right)$, then $y_{0}^{\prime}\left(t_{1}\right)<z_{0}^{\prime}\left(t_{1}\right)$ by $\left(\mathrm{i}_{0}\right)$, so that for small $t-t_{1}>0, y_{0}(t)<z_{0}(t)$. Suppose, if possible, that there exists a least $s, t_{1}<s<b$, where $y_{0}(s)=z_{0}(s)$. Then 
$y_{0}^{\prime}(s) \geqq z_{0}^{\prime}(s)$, and so, $y_{1}(s) \geqq z_{1}(s)$. This contradiction shows that $y_{0} \neq z_{0}$ on $\left(t_{1}, b\right)$. Similarly, $y_{0} \neq z_{0}$ on $\left(a, t_{1}\right)$. This proves (iii $)$.

In proving the existence of a solution of (1.4) satisfying (1.5), we can suppose that $t_{1}<t_{2}$ (as the proof for $t_{1}>t_{2}$ is similar). We can then suppose that $t_{2}=b$.

Let $S$ be the set of $y_{1}(b)$-values of solutions $y(t)$ of (1.4) satisfying $y_{0}\left(t_{1}\right)=c_{1}$. As in the last section, $S$ is a connected set, and it suffices to show that $S$ is not bounded from above or below. Suppose that $S \subset(-\infty, M)$. Let $\left\{y_{(n)}(t)\right\}$ be a sequence of solutions of (1.4) satisfying (2.2). Then, as in the last section, there exist sequences $\left\{s_{n}\right\},\left\{t_{n}\right\}$ such that $(2.5)$ holds.

It is clear from (2.2) that there exists a sequence $\left\{t_{n 1}\right\}$ such that $t_{1}<t_{n 1} \rightarrow t_{1}$ and $y_{n 1}\left(t_{n 1}\right) \rightarrow \infty$ as $n \rightarrow \infty$. Consider any solution $y=y^{*}(t)$ of (1.4) satisfying $y_{1}^{*}(b)=M$. Thus $y_{1}^{*}(b)>y_{n 1}(b)$ for all $n$, but $y_{1}^{*}\left(t_{n 1}\right)<y_{n 1}\left(t_{n 1}\right)$ for large $n$. Thus

$$
y_{1}^{*}\left(t_{n}^{\prime}\right)=y_{n 1}\left(t_{n}^{\prime}\right) \text { for some } t_{n}^{\prime}, t_{n 1}<t_{n}^{\prime}<b .
$$

Also $y_{0}^{*}\left(t_{n}\right)<y_{n 0}\left(t_{n}\right), y_{0}^{*}\left(s_{n}\right)>y_{n 0}\left(s_{n}\right)$ for large $n$, so that

$$
y_{0}^{*}\left(s_{n}^{\prime}\right)=y_{n 0}\left(s_{n}^{\prime}\right) \text { for some } s_{n}^{\prime}, s_{n}<s_{n}^{\prime}<t_{n} .
$$

The last two displays contradict (iii*) if $s_{n}^{\prime} \neq t_{n}^{\prime}$. If $s_{n}^{\prime}=t_{n}^{\prime}$ so that $y^{*}=y_{(n)}$ at $t=s_{n}^{\prime}=t_{n}^{\prime}>t_{1}$, let $y^{* *}=y_{(n)}$ for $a<t \leqq s_{n}^{\prime}$ and $y^{* *}=y^{*}$ for $s_{n}^{\prime}<t \leqq b$. Then $y^{* *}$ is a solution of (1.4) such that $y_{0}^{* *}\left(t_{1}\right)=y_{n 0}\left(t_{1}\right)=c_{1}$ and $y_{1}^{* *}(b)=y_{1}^{*}(b)=M$, so that $M \in S$. Hence, $S$ is not bounded from above. Similarly, it is not bounded from below, and Theorem I 1.2 follows.

4. Proof of Lemma I 1.1. Consider the following linear system of differential equations with continuous coefficients on an interval $J:\left|t-t_{0}\right| \leqq L$.

$$
\begin{aligned}
y_{k}^{\prime} & =\sum_{j=0}^{k+1} P_{k j}(t) y_{j} \text { for } k=0, \ldots, N-2, \\
y_{N-1}^{\prime} & =\sum_{j=0}^{N-1} P_{N-1, j}(t) y_{j}+Q(t),
\end{aligned}
$$

and $M, c>0$ constants such that

$$
\left|P_{k j}(t)\right| \leqq M, \quad P_{k, k+1}(t) \geqq c>0, \quad|Q(t)| \leqq 2 M,
$$

for all $j, k$. The corresponding homogeneous system, $Q(t) \equiv 0$, will be called the system (4.1-0).

(a) Transformation of (4.1-0). If $y=y(t)$ is a solution of (4.1-0) such that the Euclidean length $\left\|y\left(t_{0}\right)\right\|$ of $y(t)$ at $t=t_{0}$ is 1 , then a standard inequality gives

$$
\|y(t)\| \leqq \exp (L M N) \text { for }\left|t-t_{0}\right| \leqq L ;
$$

cf. [6, p. 54]. Hence

$$
\left\|y(t)-y\left(t_{0}\right)\right\| \leqq\left(L M N^{3 / 2}\right) \exp (L M N) \text { for }\left|t-t_{0}\right| \leqq L .
$$


For $i=0, \ldots, N-1$, let $y^{i}(t)=\left(y_{0 i}(t), \ldots, y_{N-1, i}(t)\right)$ be the solution of $(4.1-0)$ satisfying $y_{k i}\left(t_{0}\right)=2$ or 0 according as $k=i$ or $k \neq i$. Then (4.3) implies the existence of a constant $\delta=\delta(M, N)>0$ such that if

$$
0<L<\delta
$$

then

$$
2^{k-1} \leqq W_{k}(t) \equiv \operatorname{det}\left(y_{j i}(t)\right)_{i, j=0, \ldots, k-1} \leqq 2^{k+1} \text { for }\left|t-t_{0}\right| \leqq L
$$

and $k=0, \ldots, N-1$. Thus, $(4.1-0)$ is equivalent to an $N$ th order differential equation

$$
\left\{a_{N-1} \cdots\left[a_{1}\left(a_{0} y_{0}\right)^{\prime}\right]^{\prime} \cdots\right\}^{\prime}=0 \text {, }
$$

where the functions $a_{0}, \ldots, a_{N-1}$ are given by

$$
\begin{array}{ll}
a_{k}(t)=W_{k-1}^{2} / P_{k-1, k} W_{k-2} W_{k} & \text { for } k=0, \ldots, N-1, \\
& W_{-2}=W_{-1}=1 \text { and } P_{-1,0}=1 ;
\end{array}
$$

[6, pp. 51-54]. The system (4.1-0) and equation (4.6) are equivalent in the sense that if $y(t)=\left(y_{0}(t), \ldots, y_{N-1}(t)\right)$ is a solution of (4.1-0), then $y_{0}(t)$ is a solution of (4.6). Conversely, if $y_{0}(t)$ is a solution of (4.6), then $y=\left(y_{0}, \ldots, y_{N-1}\right)$ is a solution of $(4.1-0)$ if

$$
y_{k}=\sum_{j=0}^{k}\left(W_{j} / W_{j-1}\right) w_{j} \text { for } k=0, \ldots, N-1,
$$

(4.9) $w_{0}=a_{0} y_{0}$ and $w_{j}=a_{j}\left\{a_{j-1} \cdots\left[a_{1}\left(a_{0} y_{0}\right)^{\prime}\right]^{\prime} \cdots\right\}^{\prime} \quad$ for $j=1, \ldots, N-1$.

(The formulae (4.8), (4.9) are not stated explicitly in [6], but can be easily verified from the calculations there.)

(b) Transformation of (4.1). Correspondingly, the inhomogeneous system (4.1) is equivalent to

$$
\left\{a_{N-1} \cdots\left[a_{1}\left(a_{0} y_{0}\right)^{\prime}\right]^{\prime} \cdots\right\}^{\prime}=W_{N-2} Q / W_{N-1},
$$

by virtue of (4.8), (4.9).

(c) A determinant estimate. It is clear from (4.8) that boundary conditions of the type (1.3) are equivalent to conditions of the type

$$
w_{k}\left(t_{j}\right)=\gamma_{j k} \text { for } j=1, \ldots, m \text { and } k=0, \ldots, n(j)-1
$$

for suitable constants $\gamma_{j k}$. Let $s<t_{1}$ and consider the $N$ linearly independent solutions $y_{00}, \ldots, y_{N-1,0}$ of (4.6) given by

$$
\begin{aligned}
& y_{00}=a_{0}^{-1}(t) \text { and } \\
& y_{i 0}=a_{0}^{-1}(t) \int_{s}^{t} a_{1}^{-1}\left(r_{1}\right) d r_{1} \int_{s}^{r_{1}} a_{2}^{-1}\left(r_{2}\right) d r_{2} \cdots \int_{s}^{r_{i-1}} a_{i}^{-1}\left(r_{i}\right) d r_{i},
\end{aligned}
$$

for $i=1, \ldots, N-1$. Let $\left(w_{i 0}=a_{0} y_{i 0}, w_{i 1}, \ldots, w_{i, N-1}\right)$ belong to the solution $y_{i 0}$, by 
virtue of (4.9). We shall estimate from below the absolute value of the determinant $D$ of the matrix of coefficients of the (algebraic) linear system

$$
\sum_{i=0}^{N-1} x_{i} w_{i k}\left(t_{j}\right)=\gamma_{j k} \text { for } j=1, \ldots, m \text { and } k=0, \ldots, n(j)-1 \text {. }
$$

In fact, we shall show that there exists a constant $K>0$, depending only on $M$ and $c>0$, such that

$$
|D| \geqq K \prod_{1 \leqq j<k \leqq m}\left(t_{k}-t_{j}\right)^{n(k) n(j)} .
$$

Consider first the case $m=N, n(1)=\cdots=n(N)=1$, and denote $D$ by

$$
D_{N}=D_{N}\left(t_{1}, \ldots, t_{N}\right)=D_{N}\left(t_{1}, \ldots, t_{N} ; a_{0}, \ldots, a_{N-1}\right) ;
$$

so that

$$
\begin{aligned}
D_{N} & =\operatorname{det}\left(w_{i 0}\left(t_{j+1}\right)\right)_{i, j=0, \ldots, N-1} \\
& =\operatorname{det}\left(a_{0}\left(t_{j+1}\right) y_{i 0}\left(t_{j+1}\right)\right)_{i, j=0, \ldots, N-1} .
\end{aligned}
$$

Since $t_{k}$ occurs only in the $k$ th row, it is easy to see that

$$
\left(\partial^{N-1} / \partial t_{1} \cdots \partial t_{N-1}\right) D_{N}=a_{1}^{-1}\left(t_{1}\right) \cdots a_{1}^{-1}\left(t_{N-1}\right) D_{N-1},
$$

where $D_{N-1}=D_{N-1}\left(t_{1}, \ldots, t_{N-1}\right)=D_{N-1}\left(t_{1}, \ldots, t_{N-1} ; a_{1}, \ldots, a_{N-1}\right)$. Also $D_{N}=0$ if $t_{N}=t_{N-1}$ and $\left(\partial^{N-k} / \partial t_{k} \cdots \partial t_{N-1}\right) D_{N}=0$ if $t_{k}=t_{k-1}$ for $k=2, \ldots, N-2$. Hence

$$
\begin{aligned}
& D_{N}\left(t_{1}, \ldots, t_{N}\right)=\int_{t_{N-1}}^{t_{N}} a_{1}^{-1}\left(s_{N-1}\right) d s_{N-1} \\
& \ldots \int_{t_{2}}^{s_{3}} a_{1}^{-1}\left(s_{2}\right) d s_{2} \int_{t_{1}}^{s_{2}} a_{1}^{-1}\left(s_{1}\right) D_{N-1}\left(s_{1}, \ldots, s_{N-1}\right) d s_{1} .
\end{aligned}
$$

An induction on $N$ gives (4.14) with $m=N, n(1)=\cdots=n(N)=1$.

We now make an induction on decreasing $m$. Assume that $m \leqq N-1$ and (4.14) holds if $m$ is replaced by $m+1$. Suppose that $n(i)>1$ and let $D^{\prime}=D^{\prime}(r)$ denote the corresponding determinant with $n(i)$ replaced by $n(i)-1$ and the point $t=r$ added to the set $t_{1}, \ldots, t_{m}$, where $r>t_{i}$ is near $t_{i}$. On the one hand, the analogue of (4.14) holds for $D^{\prime}$, by the induction hypothesis, and implies that

$$
\lim _{r \rightarrow t_{i}+0} \sup \left|D^{\prime}(r)\right| /\left(r-t_{i}\right)^{n(i)-1} \geqq K \prod_{1 \leqq j<k \leqq m}\left(t_{k}-t_{j}\right)^{n(k) n(j)} .
$$

On the other hand,

$$
\left[a_{k}(r)(\partial / \partial r) \cdots a_{2}(r)(\partial / \partial r) a_{1}(r)(\partial / \partial r)\right]_{r=t_{i}} D^{\prime}(r)=0 \text { or } \quad D,
$$

according as $0 \leqq k<n(i)-1$ or $k=n(i)-1$. The last two displays imply (4.14).

(d) An a priori estimate. The fact that $D \neq 0$ implies that (4.1), (1.3) or (4.11) has a unique solution $y(t)$. We shall show that there exists a constant $C_{0}$, depending only on $M, c>0$, and $\left(t_{1}, \ldots, t_{m} ; n(1), \ldots, n(m) ; c_{0}, \ldots, c_{m, n(m)-1}\right)$, such that

$$
\|y(t)\|,\left\|y^{\prime}(t)\right\| \leqq C_{0} .
$$


To this end, let $y_{0}=\eta_{0}(t)$ be the particular solution of (4.10) given by $\eta_{0}(t)=\int_{t_{0}}^{t} a_{0}^{-1}\left(a_{N-1}\right) d s_{N-1} \cdots \int_{t_{0}}^{s_{2}} a_{0}^{-1}\left(s_{1}\right) d s_{1} \int_{t_{0}}^{s_{1}}\left[W_{N-2}\left(s_{0}\right) Q\left(s_{0}\right) / W_{N-1}\left(s_{0}\right)\right] d s_{0}$.

Then the desired solution $y(t)$ of (4.1) is obtained from (4.8), (4.9), and $y_{0}(t)=$ $\sum_{i=1}^{N-1} x_{i} y_{i 0}(t)+\eta_{0}(t)$, where the sum is a solution of the homogeneous equation (4.6) and the constants $x_{0}, \ldots, x_{N-1}$ are chosen so that

$$
\sum_{i=0}^{N-1} x_{i} y_{i k}\left(t_{j}\right)=c_{k j}-n_{k}\left(t_{j}\right)
$$

These conditions can be transformed into (4.13), where $\gamma_{k j}$ depend linearly on $c_{k j}$ with bounded coefficients. The determinant estimate (4.14) gives bounds for the constants $x_{0}, \ldots, x_{N-1}$ implying the existence of the a priori bound $C_{0}$ in (4.15).

(e) Completion of the proof. Rewrite (1.4) in the form

$$
\begin{aligned}
y_{k}^{\prime} & =\sum_{j=0}^{k+1} P_{k j}(t, y) y_{j} \text { for } k=0, \ldots, N-2, \\
y_{N-1}^{\prime} & =\sum_{j=0}^{N-1} P_{N-1, j}(t, y) y_{j}+q(t, y),
\end{aligned}
$$

where the functions $P_{N-1, j}$ and $q$ are defined by

$$
\begin{aligned}
P_{N-1, j} & =g_{N-1}(t, y) y_{j} /(1+\|y\|)^{2}, \\
q & =g_{N-1}(t, y)(1+2\|y\|) /(1+\|y\|)^{2} .
\end{aligned}
$$

Then, by (1.8)-(1.10),

$$
\left|P_{k j}(t, y)\right| \leqq M, \quad P_{k, k+1}(t, y) \geqq c>0, \quad|q(t, y)| \leqq 2 M .
$$

Suppose that $J$ is the interval $\left|t-t_{0}\right| \leqq L$ and that (4.4) holds.

In the Banach spaces $C^{0}\left(J, R^{N}\right)$ of continuous, $R^{N}$-valued functions with the sup norm on $J$, let $\Sigma$ be the set of functions $z(t)=\left(z_{0}(t), \ldots, z_{N-1}(t)\right)$ of class $C^{1}$ satisfying

$$
\|z(t)\|,\left\|z^{\prime}(t)\right\| \leqq C_{0}
$$

cf. (4.15). Consider a map $T: \Sigma \rightarrow \Sigma$, where $y(t)=T z(t)$ is the unique solution of the linear system

$$
\begin{aligned}
y_{k}^{\prime} & =\sum_{j=0}^{k+1} P_{k j}(t, z(t)) y_{j} \text { for } k=0, \ldots, N-2, \\
y_{N-1}^{\prime} & =\sum_{j=0}^{N-1} P_{N-1, j}(t, z(t)) y_{j}+q(t, z(t)),
\end{aligned}
$$


satisfying (1.3). Parts (a)-(d) on linear systems (4.1) imply the existence of such a unique solution $y(t)$, and (4.15) shows that $y \in \Sigma$. It is clear that $T$ is continuous.

Since $\Sigma$ is a compact, convex set of $C^{0}\left(J, R^{N}\right)$, the Schauder theorem implies that $T$ has a fixed point $y=y(t)$. This proves Lemma $I$ 1.1.

\section{PART II. $N$-PARAMETER FAMILIES AND PSEUdOdERIVATIVES}

1. $N$-parameter families. The following definition was given by Beckenbach [2] for $N=2$, and by Tornheim [15] for arbitrary $N$ :

Definition 1.1. $N$-PARAMETER family. Let $J^{0}$ be an open $t$-interval $(a, b)$. A set $F$ of functions $f^{0}(t) \in C^{0}\left(J^{0}\right)$ is said to be an $N$-parameter family on $J^{0}$ or to interpolate uniquely at $N$ distinct points if, for every set of $N$ distinct points $t_{1}<\cdots<t_{N}$ of $J^{0}$ and every set of $N$ numbers $c_{1}, \ldots, c_{N}$, there is one and only one element $f_{0} \in F$ satisfying

$$
f^{0}\left(t_{j}\right)=c_{j} \text { for } j=1, \ldots, N \text {, where } t_{1}<\cdots<t_{N} .
$$

The basic facts about $N$-parameter families are contained in the following lemma:

Lemma II 1.1 (BeCKenbaCh [2], ToRnheim [15]). Let $F=\left\{f^{\circ}\right\}$ be an $N$-parameter family on $J^{0}=(a, b) .(\alpha)$ If $f^{0}, g^{0}$ are distinct elements of $F$ such that $f^{0}-g^{0}$ has $N-1$ zeros on $J^{0}$, then $f^{0}-g^{0}$ changes signs at each of its zeros. $(\beta)$ If $f^{0}(t)$ $=f^{0}\left(t, t_{1}, \ldots, t_{N}, c_{1}, \ldots, c_{N}\right)$ is the unique element of $F$ determined by (1.1), then $f^{0}$ is a continuous function of its $2 N+1$ variables for $t \in J^{0}, a<t_{1}<\cdots<t_{N}<b$, $\left(c_{1}, \ldots, c_{N}\right) \in R^{N}$.

Definition 1.2. A family $F=\left\{f^{0}\right\}$ of continuous functions on an open interval $J^{0}=(a, b)$ will be said to be a local $N$-parameter family if, for every $t_{0} \in J^{0}$, there is an open interval $J\left(t^{0}\right), t_{0} \in J\left(t_{0}\right) \subset J^{0}$, such that the set of restrictions $f^{0} \mid J\left(t_{0}\right)$ of the elements $f^{0} \in F$ is an $N$-parameter family on $J\left(t_{0}\right)$.

THEOREM II 1.1. Let $J^{0}=(a, b)$ be an open interval. Let $F=\left\{f^{\circ}\right\}$ be a family of continuous functions on $J^{0}$ satisfying (i) $F$ is a local $N$-parameter family on $J^{0}$ and (ii) if $f^{0}, g^{0}$ are distinct elements of $F$, then $f^{0}-g^{0}$ has at most $N-1$ zeros on $J^{0}$. Then $F$ is an $N$-parameter family on $J^{0}$.

The proof of Theorem II 1.1 is contained in the following three propositions.

Proposition 1.1. Assume the conditions of Theorem II 1.1 and let

$$
\begin{aligned}
& \Delta=\Delta\left(J^{0}\right)=\left\{\left(t_{1}, \ldots, t_{N}\right) \in R^{N} ; a<t_{1}<\cdots<t_{N}<b\right\}, \\
& \Omega=\Omega\left(J^{0}\right)=\left\{\left(t_{1}, \ldots, t_{N}, f^{0}\left(t_{1}\right), \ldots, f^{0}\left(t_{N}\right)\right) \in \Delta \times R^{N} \subset R^{2 N}: f^{0} \in F\right\} .
\end{aligned}
$$

Then $\Omega$ is an open subset of $R^{2 N}$.

Proof. Let $t_{0} \in J$ and $s_{1}<\cdots<s_{N}$ be points of $J\left(t_{0}\right)$. Let

$$
f^{0}(t)=f^{0}\left(t, s_{1}, \ldots, s_{N}, c_{1}, \ldots, c_{N}\right)
$$


be the unique element of $F$ satisfying $f^{\circ}\left(s_{j}\right)=c_{j}$ for $j=1, \ldots, N$. For fixed $t_{0}$ and $s_{1}, \ldots, s_{N}$, consider the map $\Phi: \Delta \times R^{N} \rightarrow \Omega$ defined by

$$
\Phi\left(t_{1}, \ldots, t_{N}, c_{1}, \ldots, c_{N}\right)=\left(t_{1}, \ldots, t_{N}, f^{\circ}\left(t_{1}\right), \ldots, f^{\circ}\left(t_{N}\right)\right) .
$$

Then $\phi \in C^{0}\left(\Delta \times R^{N}\right)$, for $f^{0}\left(t, s_{1}, \ldots, s_{N}, c_{1}, \ldots, c_{N}\right)$ is a continuous function of $\left(t, c_{1}, \ldots, c_{N}\right)$ by Lemma II 1.1 applied to $J\left(t_{0}\right)$ and to small open (necessarily overlapping) $t$-intervals covering a closed subinterval of $J^{0}$. It is one-to-one by condition (ii) of Theorem II 1.1. Since the domain $\Delta \times R^{N}$ is open in $R^{2 N}$, its range $\Omega$ is open in $R^{2 N}$, by the Brouwer theorem on the invariance of domain.

The next result is a generalization of the continuity theorem (Lemma II 1.1) for $N$-parameter families.

Proposition 1.2. Let $F$ be a family of continuous functions $f^{\circ}(t)$ on an open interval $J^{0}=(a, b)$ such that $\left(\mathrm{i}^{\prime}\right)$ the set $\Omega \subset R^{2 N}$ is open and (ii) if $f^{0}, g^{0}$ are distinct elements of $F^{0}$, then $f^{0}-g^{0}$ has at most $N-1$ zeros on $J^{0}$. (a) If $f^{0}, g^{0} \in F$ and $f^{0}-g^{0}$ has exactly $N-1$ zeros on $J^{0}$, then $f^{0}-g^{0}$ changes signs at each of its zeros. (b) If $f^{0}(t)=f^{0}\left(t, t_{1}, \ldots, t_{N}, c_{1}, \ldots, c_{N}\right)$ is the unique element of $F$ satisfying (1.1), then $f^{0}$ is a continuous function of its $2 N+1$ variables for $t \in J^{0},\left(t_{1}, \ldots, t_{N}, c_{1}, \ldots, c_{N}\right) \in \Omega$.

Proof. We indicate the proof of the first part (with a slight variant of Beckenbach's proof [2] of the first part of Lemma II 1.1). Let $f^{0}, g^{0} \in F$ be distinct elements of $F$ with $f^{0}-g^{0}$ vanishing at $t=t_{1}, \ldots, t_{N-1}$. Suppose that $f^{0}-g^{0}>0$ for small $\left|t-t_{k}\right|>0$ for some $k$. Choose $s$ arbitrarily, $t_{k-1}<s<t_{k}$ if $k>1$ or $a<s<t_{k}$ if $k=1$. If $\varepsilon>0$ is sufficiently small, so that $g^{0}\left(t_{k}+\varepsilon\right)$ is sufficiently near to $f^{0}\left(t_{k}\right)=g^{0}\left(t_{k}\right)$, then the openness of $\Omega$ implies that there exists an $h^{0} \in F$ such that

$$
\begin{aligned}
h^{0}\left(t_{j}\right) & =f^{0}\left(t_{j}\right)=g^{0}\left(t_{j}\right) \text { for } j \neq k, \\
h^{0}(s) & =f^{0}(s) \text { and } h^{0}\left(t_{k}+\varepsilon\right)=g^{0}\left(t_{k}+\varepsilon\right) .
\end{aligned}
$$

Hence $f^{0}-h^{0}=0$ at the $N-1$ points $t=t_{j}, j \neq k$ and $t=s$, while $h^{0}-g^{0}=0$ at the $N-1$ points $t=t_{j}, j \neq k$, and $t=t_{k}+\varepsilon$. But it is also easy to see that either $f^{0}-h^{0}$ or $h^{0}-g^{0}$ vanishes on $\left(s, t_{k}+\varepsilon\right)$. This contradicts condition (ii) and proves the first part of Proposition 1.1.

We shall omit the proof of the second part, as it follows the proof of the last part of Lemma II 1.1 in [15].

Remark. Let $F$ satisfy (i'), (ii) of Proposition 1.2. It is tempting to try to enlarge $F$ and $\Omega$ as follows: Let $F_{0}$ be the closure of $F$ with respect to uniform convergence on compacts of $J^{0}$ and let $\Omega_{0}=\bar{\Omega} \cap\left(\Delta \times R^{N}\right)$, where $\bar{\Omega}$ is the closure of $\Omega$ in $R^{2 N}$. Unfortunately, even for $N=1$, it is easy to see that both of the following assertions $(\alpha),(\beta)$ can be false: $(\alpha)$ if $f^{0}, g^{0}$ are distinct elements of $F_{0}$, then $f^{0}-g^{0}$ has at most $N-1$ zeros and $(\beta)$ if $\left(t_{1}, \ldots, t_{N}, c_{1}, \ldots, c_{N}\right) \in \Omega_{0}$, then there is an $f^{0} \in F_{0}$ such that $f^{\circ}\left(t_{k}\right)=c_{k}$ for $k=1, \ldots, N$.

Proposition 1.3. Let $F$ be a set of continuous functions $f^{\circ}(t)$ on an open interval $J^{0}=(a, b)$. Then necessary and sufficient for $F$ to be an $N$-parameter family on $J^{0}$ is 
that it have properties (i'), (ii) of Proposition 1.2; the property (iii) if $f_{1}^{0}, f_{2}^{0}, \ldots$ is any sequence of elements of $F$ satisfying

$$
\begin{aligned}
& f_{n}^{0}(t) \leqq f_{n+1}^{0}(t) \quad \text { for } n=1,2, \ldots, \quad \text { or } \\
& f_{n}^{0}(t) \geqq f_{n+1}^{0}(t) \quad \text { for } n=1,2, \ldots,
\end{aligned}
$$

on a compact $[\alpha, \beta] \subset J^{0}$, then either

$$
f^{0}(t)=\lim _{n \rightarrow \infty} f_{n}^{0}(t) \text { exists on } J^{0} \text { and } f^{0} \in F
$$

or

$$
\lim _{n \rightarrow \infty}\left|f_{n}^{0}(t)\right|=\infty \text { on a dense set of } J^{0}
$$

and the property (iv) that, for all $t_{0} \in J^{0}$, the set $S\left(t_{0}\right)=\left\{f^{0}\left(t_{0}\right): f^{0} \in F\right\}$ is not bounded from above or below.

It is clear from Lemma II 1.1 that $F$ in Theorem II 1.1 has the properties (i'), (ii), (iii), (iv). In fact, if (1.3) does not hold, then $\left|f_{n}^{0}(t)\right| \rightarrow \infty$ as $n \rightarrow \infty$ except for at most $N-1$ points of $J^{0}$. Thus Proposition 1.3 implies Theorem II 1.1.

Proof. The necessity of (i'), (ii), (iii), (iv) is clear from Lemma II 1.1, the proof of Proposition 1.1, and from Proposition 1.2.

Assume conditions (i'), (ii), (iii), (iv). We shall show that $F$ is an $N$-parameter family, i.e., that $\Omega=\Delta \times R^{N}$. Let $k$, where $1 \leqq k \leqq N$, and $\left(t_{1}, \ldots, t_{N}\right) \in \Delta$, $\left(c_{1}, \ldots, c_{k-1}, c_{k+1}, \ldots, c_{N}\right)$ be fixed, and let $S=\left\{c_{k}:\left(t_{1}, \ldots, t_{N}, c_{1}, \ldots, c_{N}\right) \in \Omega\right\}$.

Clearly $S$ is open.

We shall show that $S$ is also closed. If $S$ is not empty, consider a sequence $\left\{c_{k n}\right\}$ satisfying $c_{k n} \in S$ for $n=1,2, \ldots$, and $c_{k n} \rightarrow c_{k}$ as $n \rightarrow \infty$. We wish to show that $c_{k} \in S$. We can suppose that $\left\{c_{k n}\right\}$ is a monotone sequence, say, for definiteness, that $c_{k n} \leqq c_{k, n+1}$ for $n=1,2, \ldots$ By Proposition 1.2, if $f_{n}^{0} \in F$ satisfies $f_{n}^{0}\left(t_{j}\right)=c_{j}$ for $j \neq k, f_{n}^{0}\left(t_{k}\right)=c_{k n}$, then

$$
\begin{aligned}
(-1)^{i+k-1} f_{n}^{0}(t) & \leqq(-1)^{i+k-1} f_{n+1}^{0}(t) & & \text { for } t \in\left(t_{i}, t_{i+1}\right), i=0, \ldots, k-2, \\
f_{n}^{0}(t) & \leqq f_{n+1}^{0}(t) & & \text { for } t \in\left(t_{k-1}, t_{k+1}\right), \\
(-1)^{i+k} f_{n}^{0}(t) & \leqq(-1)^{i+k} f_{n+1}^{0}(t) & & \text { for } t \in\left(t_{i}, t_{i+1}\right), i=k+1, \ldots, N,
\end{aligned}
$$

where $a=t_{0}$ and $b=t_{N+1}$. Thus, by condition (iii), either (1.3) holds and $c_{k} \in S$ or (1.4) holds.

In the latter case, choose an arbitrary $f^{0} \in F$ satisfying $f^{0}\left(t_{k}\right)>c_{k}>f_{n}^{0}\left(t_{k}\right)$ for $n=1,2, \ldots$; cf. condition (iv). Then, for large $n, f^{0}-f_{n}^{0}$ vanishes on either side of $t_{k}$ arbitrarily near $t_{k}$, and also arbitrarily near $t_{j}, j \neq k$. Thus, for large $n, f^{0}-f_{n}^{0} \not \equiv 0$ has $N$ zeros on $J^{0}$. This contradiction shows that (1.4) cannot hold. Thus $S$ is closed. Hence $S=\varnothing$ or $S=(-\infty, \infty)$. Clearly $\Omega(a, b)=\Delta(a, b) \times R^{N}$. This proves Proposition 1.3.

It will be useful below to have the following remark. 
Proposition 1.4. Let $G\left(t, x_{1}, \ldots, x_{N}\right)$ be continuous on $J^{0} \times X$, where $J^{0}$ $=(a, b), X \subset R^{N}$ is open. Let $F$ be the family of functions $f^{0}(t)=G\left(t, x_{1}, \ldots, x_{N}\right)$ on $J^{0}$, for fixed $\left(x_{1}, \ldots, x_{N}\right)$. Let $F$ have the properties (ii), (iii), (iv) of Proposition 1.3. Then $F$ is an $N$-parameter family on $J^{0}$.

In fact, that $F$ has the property (i') is clear from the proof of Proposition 1.1.

Under additional conditions, we can get an analogue of Theorem II 1.1 on halfopen intervals.

Corollary II 1.1. Let $N>1$ and $J=(a, b]$ be a half-open interval. Let $F$ be $a$ family of continuous functions on $J$ such that their restrictions to $J^{0}=(a, b)$ is an $N$-parameter family on $J^{0}$ (e.g., let their restrictions satisfy (i), (ii) of Theorem II 1.1). In addition, suppose that $\left(\mathrm{iv}_{b}\right)$ the set of numbers $\left\{f^{0}(b): f^{0} \in F\right\}$ is not bounded from above or below and $(\mathrm{v})$ if $f^{0}$ and $f_{1}^{0}, f_{2}^{0}, \ldots$ are elements of $F$ satisfying (1.3), then $f_{n}^{0}(b) \rightarrow f^{0}(b)$ as $n \rightarrow \infty$. Then, for arbitrary points $t_{1}<\cdots<t_{N}$ of $J$ and arbitrary $c_{1}, \ldots, c_{N}$, there exists at least one $f^{0} \in F$ satisfying (1.1).

Proof. Only the case $t_{N}=b$ need be considered. Let $f_{c}^{0}(t) \in F$ be the unique element of $F$ satisfying

$$
f_{c}^{0}\left(t_{j}\right)=c_{j} \text { for } j \neq N \text { and } f_{c}^{0}(s)=c,
$$

where $s$ is fixed on $t_{N-1}<s<b$. Let $S=\left\{f_{c}^{0}(b):-\infty<c<\infty\right\}$. It suffices to show that $S=(-\infty, \infty)$.

By Lemma II 1.1, the function $f_{c}^{0}(t)$ is continuous for $t \in J^{0},-\infty<c<\infty$. By condition ( $v$ ) of the corollary, it is also continuous for $t \in J,-\infty<c<\infty$. For by Lemma II 1.1 , if $c<d$, then

$$
(-1)^{N-1+i} f_{c}^{0}<(-1)^{N-1+i} f_{d}^{0} \text { on }\left(t_{i}, t_{i+1}\right) \text { for } i=0, \ldots, N-1,
$$

where $t_{0}=a$. Thus $S$ is connected.

It suffices now to show that $S$ is not bounded from either above or below. But this can be done by the arguments used in the last part of the proof of Proposition 1.3.

Definition 1.3. Pseudoderivatives. Let $\mathscr{F}$ be a family of $R^{K}$-valued functions $f(t)=\left(f^{0}(t), \ldots, f^{K-1}(t)\right)$ defined on an open interval $J^{0}$ and let $f^{0}(t) \in C^{0}\left(J^{0}\right)$. Let $F$ denote the family of continuous functions $F=\left\{f^{0}:\left(f^{0}, \ldots, f^{K-1}\right) \in \mathscr{F}\right\}$. Suppose that for every $t_{0} \in J^{0}$ and every set of $K$ numbers $c_{00}, \ldots, c_{0, K-1}$, there is at least one $f \in \mathscr{F}$ such that

$$
f^{k}\left(t_{0}\right)=c_{0 k} \quad \text { for } k=0, \ldots, K-1 .
$$

Also, for $f^{0}, g^{0} \in F$, suppose that

$$
\left(t-t_{0}\right)^{t}\left[f^{0}(t)-g^{0}(t)\right]>0 \text { for small }\left|t-t_{0}\right|>0
$$

whenever

$$
\begin{array}{cl}
f^{i}\left(t_{0}\right)=g^{i}\left(t_{0}\right) & \text { for } i=0, \ldots, I-1(\leqq K-2), \\
& f^{I}\left(t_{0}\right)>g^{I}\left(t_{0}\right) .
\end{array}
$$


This situation will be described by saying that the elements $f^{0} \in F$ have $K-1$ pseudoderivatives $\left(f^{1}\left(t_{0}\right), \ldots, f^{K-1}\left(t_{0}\right)\right)$ at $t=t_{0}$, and the function $f^{i}=f^{i}(t)$ will be called the $i$ th pseudoderivative of $f^{0}$.

Remark 1. Note that, in this definition, $f^{i}\left(t_{0}\right)$ is a fixed functional $\left(t_{0}, f^{0}\right) \mapsto$ $f^{i}\left(t_{0}\right)$ of $t_{0} \in J^{0}, f^{0} \in F$. There is no assumption that $F$ is a linear set nor that there is any linearity in the map $f^{0} \mapsto f^{i}$. Nor is there any assumption of continuity for the map $t \mapsto f^{i}(t)$. Even if $f^{0}, \ldots, f^{K-1}$ are continuous functions of $t$, the functions $f^{2}, \ldots, f^{K-1}$ need not be pseudoderivatives of $f^{1}$ and, of course, it is meaningless to speak of a "product rule."

REMARK 2. Definition 1.3 implies the following converse for the property “(1.7), (1.8) $\Rightarrow(1.6) ":$ If (1.7) holds and

$$
\left(t-t_{0}\right)^{I}\left[f^{0}(t)-g^{0}(t)\right]>0 \text { for either small } t-t_{0}>0 \text { or } t_{0}-t>0
$$

then

$$
f^{I}\left(t_{0}\right) \geqq g^{I}\left(t_{0}\right) .
$$

REMARK 3. The defining properties of pseudoderivatives depend on the differences $f^{0}(t)-g^{0}(t), \ldots, f^{K-1}(t)-g^{K-1}(t)$. Hence, if $c_{0}(t)>0, c(t)>0, h_{0}(t), \ldots$, $h_{K-1}(t)$ are arbitrary functions on $J$, with $c_{0}(t), h_{0}(t)$ continuous, then the functions $c_{0}(t) f^{0}(t)+h_{0}(t)$ have the pseudoderivatives $c(t) f^{1}(t)+h_{1}(t), \ldots, c(t) f^{K-1}(t)+$ $h_{K-1}(t)$. See also $\$ 3$ below.

Definition 1.4. A family $F$ of functions $f^{0}$ with $N-1$ pseudoderivatives $f^{1}, \ldots, f^{N-1}$ on $J^{0}$ as in Definition $1.3, K=N$, is said to

(i) interpolate uniquely at $N$ coincident points or to have the property of unique initial values if, for every $t_{0} \in J^{0}$ and every set of $N$ numbers $c_{00}, \ldots, c_{0, N-1}$, there is one and only one $f^{0} \in F$ such that

$$
f^{k}\left(t_{0}\right)=c_{0 k} \text { for } k=0, \ldots, N-1 ;
$$

(ii) interpolate uniquely at $N$ arbitrary points if, for any integer $m, 1 \leqq m \leqq N$, any points $t_{1}<\cdots<t_{m}$ of $J^{0}$, positive integers $n(1), \ldots, n(m)$ satisfying $n(1)+\cdots+n(m)$ $=N$, and $N$ numbers $c_{j k}$, where $j=1, \ldots, m$ and $k=0, \ldots, n(j)-1$, there is one and only one $f^{0} \in F$ such that

$$
f^{k}\left(t_{j}\right)=c_{j k} \text { for } j=1, \ldots, m \text { and } k=0, \ldots, n(j)-1 .
$$

THEOREM II $1.2(\alpha)$. Let $1 \leqq K \leqq N$ and $F$ be a family of continuous functions $f^{0}$ with $K-1$ pseudoderivatives $f^{1}, \ldots, f^{K-1}$ on an open interval $J^{0}$. Suppose that $F$ interpolates uniquely at $N$ distinct points (i.e., that $F$ is an $N$-parameter family on $J^{0}$ ). Then, for every choice of $t_{1}<\cdots<t_{m}$ on $J^{0},(n(1), \ldots, n(m))$ with $1 \leqq n(j) \leqq K$ and $n(1)+\cdots+n(m)=N$, and $N$ constants $\left(c_{10}, \ldots, c_{m, n(m)-1}\right)$, there exists at least one $f \in F$ satisfying (1.12). ( $\beta$ ) If, in addition, $K=N$ and $F$ interpolates uniquely at $N$ coincident points (i.e., $F$ has the property of unique initial values), then $F$ interpolates uniquely at $N$ arbitrary points. 
This was stated in Hartman [5] for the case that $f^{0} \in C^{N-1}\left(J^{0}\right)$ and $f^{k}=d^{k} f^{0} / d t^{k}$; cf. Theorem (I) and Lemmas 7, 8. (For a related result on linear families of solutions of an $n$th order linear differential equation, cf. [1] and [12].) Actually the proof in [5] was given for the case that $f^{1}, \ldots, f^{K-1}$ are the pseudoderivatives of Example 1 of the next section. The same proof is valid whenever $f^{1}, \ldots, f^{K-1}$ are pseudoderivatives in the sense of Definition 1.3.

In order to extend the last result to half-open intervals, we need another result of [5]. Consider a set of conditions (1.12) and divide the set of integers $j=1, \ldots, m$ arbitrarily into two sets $\left\{j^{\prime}\right\},\left\{j^{\prime \prime}\right\}$, where $1 \leqq n\left(j^{\prime}\right) \leqq K$, but $n\left(j^{\prime \prime}\right)=1$. In the next lemma, we consider the partial data $\left\{t_{j^{\prime}}, n\left(j^{\prime}\right), c_{j^{\prime} k}\right\}$ to be fixed, while $\left\{t_{j^{\prime \prime}}, n\left(j^{\prime \prime}\right)\right.$ $\left.=1, c_{j^{\prime \prime} 0}\right\}$ is arbitrary, with all $t_{j^{\prime}}, t_{j^{\prime \prime}}$ distinct (not necessarily $t_{1}<\cdots<t_{m}$ ).

LEMMA II 1.2. Let $F$ satisfy the conditions of Theorem II 1.2( $\alpha)$. Then there exists a subfamily $F^{\prime}=F^{\prime}\left\{t_{j^{\prime}}, n\left(j^{\prime}\right), c_{j^{\prime} k}\right\}$ of $F$ with the following properties:

(i) if $f^{0} \in F^{\prime}$, then $f^{0}$ satisfies the partial set of conditions

$$
f^{k}\left(t_{j^{\prime}}\right)=c_{j^{\prime} k} \text { for all } j^{\prime} \text { and } k=0, \ldots, n\left(j^{\prime}\right)-1 ;
$$

(ii) there exists a unique $f^{0} \in F^{\prime}$ satisfying (1.12) as in Theorem II 1.2( $\left.\alpha\right)$; and

(iii) if $f^{0}, g^{0}$ are distinct elements of $F^{\prime}$, then $f^{0}-g^{0}$ has at most $N-\sum n\left(j^{\prime}\right)-1$ zeros $\neq t_{j^{\prime}}$, and if $f^{0}-g^{0}$ has exactly $N-\sum n\left(j^{\prime}\right)-1$ zeros $\neq t_{j^{\prime}}$, then $f^{0}-g^{0}$ changes signs at each of these zeros and $\left(t-t_{j^{\prime}}\right)^{n\left(j^{\prime}\right)}\left[f^{0}(t)-g^{0}(t)\right]$ is of the same sign for small $\left|t-t_{j^{\prime}}\right|>0$.

The families $F^{\prime}$ can be chosen to be decreasing if either an $n\left(j^{\prime}\right)$ is increased or the set $\left\{t_{j^{\prime}}\right\}$ enlarged, and additional conditions added to (1.14). (When the uniqueness part $(\beta)$ of Theorem II 1.2 holds, then $F^{\prime}$ is merely the set of all $f^{0} \in F$ satisfying (1.14).)

For a proof, see Lemmas 3, 5 and 7 of [5] (also Lemmas 4, 6, 8 for the parenthetical part).

COROLLARY II 1.2. Let $F$ be a family of continuous functions $f^{0}$ on a half-open interval $J=(a, b]$ having $K-1$ pseudoderivatives $f^{1}, \ldots, f^{K-1}$ on $J^{0}=(a, b)$. Let $F$ satisfy the conditions of Theorem II $1.2(\alpha)$ on $J^{0}$ and conditions $\left(\mathrm{iv}_{b}\right)$, (v) of Corollary II 1.1. Then there exists an $f^{0} \in F$ satisfying (1.12) with $t_{1}, \ldots, t_{m} \in J, 1 \leqq n(j) \leqq K$, $n(1)+\cdots+n(m)=N$, provided that $t_{m}<b$ or that $t_{m}=b, n(m)=1$.

Proof. In view of Theorem II 1.2( $\alpha)$, we need consider only the case $t_{m}=b, n(m)$ $=1$. The corollary is correct if $m=N$ (so that $m(j)=1$ for $j=1, \ldots, N$ ) by Corollary II 1.1. Assume that $h<N$; that the corollary is correct if there are at least $h+1$ points $t_{j^{\prime \prime}}$ for which $n\left(j^{\prime \prime}\right)=1$; and that in this case, $f^{0}$ can be chosen in $F^{\prime}=F^{\prime}\left\{t_{j^{\prime}}, n\left(j^{\prime}\right), c_{j^{\prime} k}\right\}$.

Consider a given set of conditions (1.12) involving $h$ points $t_{j^{\prime \prime}}$ for which $n\left(j^{\prime \prime}\right)=1$, and let $i$ be the largest in the set $\left\{j^{\prime}\right\}$ with $n\left(j^{\prime}\right)>1$. Let $F^{\prime}$ be the family belonging to $t_{j^{\prime}}, n\left(j^{\prime}\right), c_{j^{\prime} k}$, and $F^{\prime \prime}$ the corresponding family with $n(i)$ replaced by $n(i)-2$ if $n(i)>2$ or with $t_{i}$ deleted from $\left\{t_{j^{\prime}}\right\}$ if $n(i)=2$. We can also suppose that $F^{\prime} \subset F^{\prime \prime}$. By 
Lemma II 1.2(iii), if $f^{0}, g^{0}$ are distinct elements of $F^{\prime \prime}$, then $f^{0}-g^{0}$ has at most $N-\left[\sum n\left(j^{\prime}\right)-2\right]-1$ zeros $\neq t_{j^{\prime}}$.

Let $t_{m-1}<s<t_{m}=b$ and let $f_{c}^{0}$ be the unique element of $F^{\prime}$, supplied by Theorem II $1.2(\alpha)$ and Lemma II 1.2, satisfying (1.12) with the condition $f^{\circ}(b)=c_{N}$ replaced by $f^{\circ}(s)=c$. Then, by Lemma II $1.2($ iii),

$$
f_{c}^{0}(t) \text { increases with } c \text { on }\left(t_{m-1}, t_{m}\right)
$$

and, for $j=1, \ldots, m$,

$$
(-1)^{n(j)+\cdots+n(m)} f_{c}^{0}(t) \text { increases with } c \text { on }\left(t_{j-1}, t_{j}\right)
$$

where $t_{0}=a$. By Lemma II 1.1( $\left.\beta\right)$, we have, except possibly at $N-m+1$ points,

$$
\begin{gathered}
f_{c}^{0}(t) \rightarrow \pm \infty \quad \text { as } c \rightarrow \infty \text { on }\left(t_{m-1}, t_{m}\right), \\
(-1)^{n(j)+\cdots+n(m)} f_{c}^{0}(t) \rightarrow \pm \infty \text { as } c \rightarrow \pm \infty \text { on }\left(t_{j-1}, t_{j}\right) .
\end{gathered}
$$

Let $S=\left\{f_{c}^{0}(b):-\infty<c<\infty\right\}$ and suppose, if possible, that $S$ is bounded from above, say, $S \subset\left(-\infty, c_{N}\right)$. By the induction hypotheses (on $h$ ), there exist elements $f^{0} \in F^{\prime \prime}$ satisfying (1.12), except the two cases $j=i, k=n(i)-1$ and $k=n(i)-2$, but satisfying

$$
(-1)^{n(i)+\cdots+n(m)}\left[f^{n(i)-2}\left(t_{i}\right)-f_{c}^{n(i)-2}\left(t_{i}\right)\right]>0,
$$

where $f_{c}^{1}, \ldots, f_{c}^{N-1}$ are the pseudoderivatives of $f_{c}^{0}$ (in fact, we could also specify $f^{0}$ at some point $\left.t \neq t_{1}, \ldots, t_{m}\right)$.

Thus, (1.19) and the definition of pseudoderivatives imply that, for any $c$,

$$
(-1)^{n(i+1)+\cdots+n(m)}\left(t-t_{i}\right)^{n(i)-1}\left[f^{0}(t)-f_{c}^{0}(t)\right]>0
$$

for small $\left|t-t_{i}\right| \neq 0$. But (1.17), (1.18) show that, for some $t$ near $t_{i}$, and on either side of $t_{i}$, the reverse inequality holds for large $c$. Thus, for large $c, f^{0}-f_{c}^{0}$ has a zero on each of the intervals $\left(t_{i}-\varepsilon, t_{i}\right),\left(t_{i}, t_{i}+\varepsilon\right)$ if $\varepsilon>0$. Also $f^{0}-f_{c}^{0}$ has a zero, for large $c$, on $\left(s, t_{m}\right)=(s, b)$.

The $t$-values in the definition of $F^{\prime \prime}$ (which are the $t_{j}$, including $t_{i}$ or not, according as $n(i)>2$ or $n(i)=2$ ) have total assigned multiplicities $\sum n\left(j^{\prime}\right)-2$. Apart from these $t$-values, $f^{0}-f_{c}^{0}$ has $\sum n\left(j^{\prime \prime}\right)-1+3$ zeros at points $t_{j^{\prime \prime}} \neq b$ and 3 other points. Since $\sum n\left(j^{\prime \prime}\right)+2=N-\left[\sum n\left(j^{\prime}\right)-2\right]$, this contradicts Lemma II 1.2(iii) applied to the family $F^{\prime \prime}$ and $f^{0}, g^{0}=f_{c}^{0} \in F^{\prime \prime}$. Hence $S$ is not bounded from above. Similarly, it is not bounded from below.

Finally, it can be seen that $S$ is connected, as in the proof of Theorem II 1.1 and Corollary II 1.1. Hence $S=(-\infty, \infty)$. This proves Corollary II 1.2 for the case of $h$ points $\left\{t_{j^{\prime \prime}}\right\}$.

In the next theorem, we shall use the notion of the continuous dependence on initial conditions.

THEOREM II 1.3. Let $F$ be a family of continuous functions $f^{\circ}(t)$ having $N-1$ pseudoderivatives $f^{1}, \ldots, f^{N-1}$ on an open interval $J^{0}=(a, b)$ such that (i) $F$ has the 
property of unique initial values and if $f^{0}(t)=f^{0}\left(t, t_{0}, c_{00}, \ldots, c_{0, N-1}\right)$ is the unique element of $F$ satisfying (1.11), then for some fixed $t_{0} \in J^{0}, f^{0}$ is continuous for $\left(t, c_{00}, \ldots, c_{0, N-1}\right) \in J^{0} \times R^{N}$; (ii) if $f^{0}, g^{0}$ are distinct elements of $F$, then $f^{0}-g^{0}$ has at most $N-1$ distinct zeros on $J^{0}$; properties (iii), (iv) of Proposition 1.3 hold. Then $F$ interpolates uniquely at $N$ arbitrary points of $J^{0}$.

In fact, $F$ is an $N$-parameter family on $J^{0}$, by Proposition 1.4, with $G\left(t, x_{1}, \ldots, x_{N}\right)=f^{0}\left(t, t_{0}, x_{1}, \ldots, x_{N}\right)$. Thus Theorem II 1.3 is a consequence of Theorem II 1.2.

2. Pseudoderivatives. In this section, we shall illustrate the concept of pseudoderivatives by a number of examples of interest.

EXAMPLE 1. Let $F$ be a family of continuous functions $f^{\circ}(t)$ on an open interval $J^{0}$ with the property that, for every $t_{0} \in J^{0}$, there are $K-1$ numbers $f^{1}\left(t_{0}\right), \ldots, f^{K-1}\left(t_{0}\right)$ such that

(2.1) $f^{0}(t)=f^{0}\left(t_{0}\right)+\left(t-t_{0}\right) f^{1}\left(t_{0}\right)+\cdots+\left(t-t_{0}\right)^{K-1} f^{K-1}\left(t_{0}\right) /(K-1) !+o\left(\left|t-t_{0}\right|^{K-1}\right)$,

as $t \rightarrow t_{0}$. Then $f^{0}$ has the pseudoderivatives $f^{1}\left(t_{0}\right), \ldots, f^{K-1}\left(t_{0}\right)$ at $t=t_{0}$.

EXAMPLE 2. Let $a_{0}(t), \ldots, a_{N-1}(t)$ be positive continuous functions on $J^{0}=(a, b)$ and let $F$ be the family of solutions $u=f^{\circ}(t)$ of the $N$ th order linear differential equation

$$
\left\{a_{N-1} \cdots\left[a_{1}\left(a_{0} u\right)^{\prime}\right] \cdots\right\}^{\prime}=0 .
$$

Then $u=f^{0}(t) \in F$ has the $N-1$ pseudoderivatives $f^{1}=\left(a_{0} f^{0}\right)^{\prime}, f^{2}=\left[a_{1}\left(a_{0} f^{0}\right)^{\prime}\right]^{\prime}, \ldots$

EXAMPLE 2a. Consider the system of linear differential equations

$$
\begin{aligned}
y_{i}^{\prime} & =\sum_{j=0}^{i+1} a_{i j}(t) y_{j} \text { for } i=0, \ldots, N-2, \\
y_{N-1}^{\prime} & =\sum_{j=0}^{N-1} a_{N-1, j}(t) y_{j},
\end{aligned}
$$

with continuous coefficients on $J^{0}=(a, b)$ and $a_{i, i+1}(t)>0$ on $(a, b)$ for $i=0, \ldots, N-2$. Let $a<t_{0}<b$. Then for every sufficiently small interval $(\alpha, \beta)$, $t_{0} \in(\alpha, \beta) \subset(a, b)$, the system (2.3)-(2.4) is equivalent to an equation (2.2) for $u=y_{0}$ (with continuous positive coefficients $a_{0}, \ldots, a_{N-1}$ defined in terms of a fundamental matrix for (2.3)-(2.4)); see Hartman [6, pp. 51-54]. Thus the conclusion of Example 2 is applicable on small $(\alpha, \beta)$.

Example 3. Consider again the system (2.3)-(2.4) and let $\mathscr{F}$ be the family of solution vectors $\left(y_{0}(t), \ldots, y_{N-1}(t)\right)$ and let $F$ be the corresponding family of functions $y_{0}(t)$. Then $y_{0}(t) \in F$ has the pseudoderivatives $y_{1}(t), \ldots, y_{N-1}(t)$ on $(a, b)$.

This is a consequence of the following:

Proposition 2.1. Let $1 \leqq K \leqq N$; and let $g_{i}\left(t, y_{0}, \ldots, y_{i+1}\right)$ for $i=0, \ldots, K-2$ and $g_{K-1}\left(t, y_{0}, \ldots, y_{N-1}\right), \ldots, g_{N-1}\left(t, y_{0}, \ldots, y_{N-1}\right)$ be continuous for $a<t<b$ and 
arbitrary $y=\left(y_{0}, y_{1}, \ldots, y_{N-1}\right)$, such that solutions of the system of differential equations

$$
\begin{aligned}
y_{i}^{\prime}=g_{i}\left(t, y_{0}, \ldots, y_{i+1}\right) & \text { for } i=0, \ldots, K-2, \\
y_{i}^{\prime}=g_{i}\left(t, y_{0}, \ldots, y_{N-1}\right) & \text { for } i=K-1, \ldots, N-1,
\end{aligned}
$$

exist on $(a, b)$. In addition, assume

$$
g_{i} \text { is locally, uniformly Lipschitz in } y \text { for } i=0, \ldots, K-3 \text {, }
$$

$$
g_{i} \text { is increasing in } y_{i+1} \text { for } i=0, \ldots, K-2 \text {. }
$$

Let $F$ be the family of functions $\left\{y_{0}(t)\right\}$, where $y=\left(y_{0}(t), \ldots, y_{N-1}(t)\right)$ is a solution of (2.5)-(2.6). Then $y_{0} \in F$ has the pseudoderivatives $y_{1}, \ldots, y_{K-1}$ on $(a, b)$.

REMARK. If $K=2$, condition (2.7) is vacuous. If $K=3$, condition (2.7) can be replaced by the condition that the (scalar) initial value problem $u^{\prime}=g_{0}\left(t, u, y_{1}(t)\right)$, $u\left(t_{0}\right)=y_{0}\left(t_{0}\right)$ has the unique solution $u=y_{0}(t)$ whenever $y=\left(y_{0}(t), y_{1}(t), \ldots, y_{N-1}(t)\right)$ is a solution of (2.5)-(2.6). As for $K \geqq 4$, see Proposition 2.3.

Proof. Let $y=\left(y_{0}(t), \ldots, y_{N-1}(t)\right)$ and $z=\left(z_{0}(t), \ldots, z_{N-1}(t)\right)$ be two solutions of (2.5)-(2.6). Let $a<t_{0}<b$ and suppose that

$$
y_{i}\left(t_{0}\right)=z_{i}\left(t_{0}\right) \text { for } i=0, \ldots, I-1(\leqq K-2), y_{I}\left(t_{0}\right)>z_{I}\left(t_{0}\right) .
$$

Let $a<\alpha<t_{0}<\beta<b ;\left|y_{j}(t)\right|,\left|z_{j}(t)\right| \leqq M$ for $\alpha \leqq t \leqq \beta ;$ and

$$
\begin{aligned}
& \left|g_{i}\left(t, u_{0}, \ldots, u_{i+1}\right)-g_{i}\left(t, v_{0}, \ldots, v_{i+1}\right)\right| \leqq L \sum_{j=0}^{i+1}\left|u_{j}-v_{j}\right| \\
& \quad \text { for } i=0, \ldots, K-3 ;\left|u_{j}\right|,\left|v_{j}\right| \leqq M ; \text { and } \alpha \leqq t \leqq \beta .
\end{aligned}
$$

To simplify the typography, let $t_{0}=0$. Below we shall consider only small $t \geqq 0$; the desired inequalities for $t<0$ are proved in the same way.

In view of (2.5), (2.8) and (2.9), $y_{1-1}^{\prime}-z_{1-1}^{\prime}>0$ at $t=0$ and hence for small $t>0$, so that $y_{I-1}-z_{I-1}>0$ for small $t>0$. Assume that

$$
y_{i+1}(t)>z_{i+1}(t) \text { for small } t>0
$$

holds for some $i, 0 \leqq i \leqq I-2(\leqq K-3)$. It will be verified that $\left(2.11_{i-1}\right)$ holds. Put

$$
h_{i}(t)=g_{i}\left(t, y_{0}(t), \ldots, y_{i}(t), y_{i+1}(t)\right)-g_{i}\left(t, y_{0}(t), \ldots, y_{i}(t), z_{i+1}(t)\right),
$$

so that $h_{i}(t)>0$ for small $t>0$. By (2.5) and (2.10),

$$
\sum_{j=0}^{i}\left|y_{j}^{\prime}-z_{j}^{\prime}\right| \leqq(i+1) L \sum_{j=0}^{i}\left|y_{j}-z_{j}\right|+h_{i}(t)
$$

and so, for $C=\exp [(i+1) L(\beta-\alpha)], \sum_{j=0}^{i}\left|y_{j}-z_{j}\right| \leqq C \int_{0}^{t} h_{i}(s) d s$. Also, (2.5) and (2.10) give

$$
y_{i}^{\prime}-z_{i}^{\prime} \geqq-L \sum_{j=0}^{i}\left|y_{j}-z_{j}\right|+h_{i}(t) \geqq-L C \int_{0}^{t} h_{i}(s) d s+h_{i}(t) .
$$


Since $h_{i}>0$ for small $t>0$,

$$
y_{i}(t)-z_{i}(t) \geqq-L C t \int_{0}^{t} h_{i}(s) d s+\int_{0}^{t} h_{i}(s) d s>0 .
$$

This implies $\left(2.11_{i-1}\right)$ and completes the proof.

Proposition 2.2. Let the assumptions of Proposition 2.1, except that involving (2.7), hold. In place of (2.7), assume

$$
g_{i} \text { is nondecreasing in } y_{j}, j \neq i, i=0, \ldots, K-3 .
$$

For $a<t_{0}<b, I=2, \ldots, K-1$, and an arbitrary solution $z=\left(z_{0}(t), \ldots, z_{N-1}(t)\right)$ of (2.5)-(2.6), assume that the initial value problem

$$
\begin{aligned}
u_{i}^{\prime} & =g_{i}\left(t, u_{0}, \ldots, u_{i+1}\right) \text { for } i=0, \ldots, I-3, \\
u_{I-2}^{\prime} & =g_{I-2}\left(t, u_{0}, \ldots, u_{I-2}, z_{I-1}(t)\right)
\end{aligned}
$$

and

$$
u_{i}\left(t_{0}\right)=z_{i}\left(t_{0}\right) \text { for } i=0, \ldots, I-2
$$

has the unique solution $u=\left(z_{0}(t), \ldots, z_{I-2}(t)\right)$. Then the conclusion of Proposition 2.1 is valid.

Proof. Let $y=\left(y_{0}(t), \ldots, y_{N-1}(t)\right)$ and $z=\left(z_{0}(t), \ldots, z_{N-1}(t)\right)$ be two solutions of (2.5)-(2.6), and let (2.9) hold. By (2.8) with $i=I-1, y_{I-1}^{\prime}\left(t_{0}\right)>z_{I-1}^{\prime}\left(t_{0}\right)$. Hence $y_{I-1}(t)>z_{I-1}(t)$ for small $t-t_{0}>0$. Thus

$$
\begin{aligned}
y_{I}^{\prime} & =g_{i}\left(t, y_{0}, \ldots, y_{i+1}\right) \quad \text { for } i=0, \ldots, I-3, \\
y_{I-2}^{\prime} & >g_{I-2}\left(t, y_{0}, \ldots, y_{I-2}, z_{I-1}(t)\right),
\end{aligned}
$$

for small $t>0$. Since $u=\left(y_{0}(t), \ldots, y_{I-2}(t)\right)$ satisfies the initial conditions (2.16), it follows from a theorem of Kamke [7] and the uniqueness of the solution of (2.15)-(2.16) that $y_{i}(t) \geqq z_{i}(t)$ for small $t-t_{0} \geqq 0$ and $i=0, \ldots, I-2$. It is then easy to see from (2.8), (2.14) and (2.17) that $y_{I-2}(t)>z_{I-2}(t)$ for small $t-t_{0}>0$. This argument can be repeated to conclude that $y_{0}(t)>z_{0}(t)$ for small $t-t_{0}>0$.

Similarly, it is shown that $\left(t-t_{0}\right)^{I}\left[y_{0}(t)-z_{0}(t)\right]>0$ for small $t_{0}-t>0$. Thus $y_{1}, \ldots y_{K-1}$ are pseudoderivatives of $y_{0}$, and the proof is complete.

Note that condition (2.7) in Proposition 2.1 is vacuous if $K<3$ and, when (2.8) holds, condition (2.14) in Proposition 2.2 is vacuous if $K<4$. It will now be shown that if $K=N \geqq 4$ and (2.7), (2.14) are omitted, then Propositions 2.1, 2.2 are false, cf. the remark following Proposition 2.1.

Proposition 2.3. Let $K=N \geqq 4$. Assume the conditions of Propositions 2.1 and 2.2, except for (2.7) and (2.14). Assume also that solutions of (2.5)-(2.6) are uniquely determined by initial conditions. Then the conclusion of Proposition 2.1 need not hold.

It will follow from the proof that, for example, the Lipschitz condition (2.7) in Proposition 2.1 cannot be replaced by the type of inequality occurring in Kamke's 
or even Osgood's uniqueness theorem. The papers M. M. Peixoto [13] and M. C. Peixoto [14] suggest that the example proving Proposition 2.3 cannot exist if the family $F=\left\{y_{0}(t)\right\}$ is an $N$-parameter family. The proof of Proposition 2.3 will depend on the following:

Lemma. On an interval $[0, b]$, there exist functions $h(t), U(t)$ with the properties: (i) $h(t) \in C^{0}[0, b], h(t) \geqq 0$ according as $t \geqq 0$; (ii) $U(t) \in C^{2}[0, b], U(t)$ changes signs for arbitrarily small $t,|U| \leqq 1$, and $U(t)$ is a solution of the initial value problem

$$
\begin{aligned}
U^{\prime \prime} & =|U| \log |U|+h(t), \\
U(0) & =U^{\prime}(0)=0 .
\end{aligned}
$$

The proof of Proposition 2.1 shows that the lemma is false if $\log |U|$ is omitted in (2.18). Assuming the lemma, we shall first prove Proposition 2.3.

Proof of Proposition 2.3. In what follows, let $b, U(t)$ and $h(t)$ be the data supplied by the lemma.

It will be shown that, for a suitable choice of the function $q(t, u, w)$, the system of differential equations

$$
u^{\prime}=v, \quad v^{\prime}=q(t, u, w), \quad w^{\prime}=x-u, \quad x^{\prime}=v
$$

has the desired properties, if $(u, v, w, x)=\left(y_{0}, y_{1}, y_{2}, y_{3}\right)$. By (2.20), we have

$$
x-u=c \text { and } w=c t+c_{0},
$$

where $c, c_{0}$ are integration constants. Hence (2.20) is equivalent to

$$
u^{\prime \prime}=q\left(t, u, c t+c_{0}\right)
$$

The function $q$ will be chosen so that

(a) $q$ is continuous and increasing in $w$ for fixed $(t, u)$;

(b) for all $\left(c, c_{0}\right)$, the solutions of (2.22) are uniquely determined by initial conditions;

(c) as a function of $u$ for bounded $(t, w), q$ is bounded; finally

(d) $q$ satisfies the relations

$$
\begin{gathered}
q(t, 0,0)=0, \\
q(t, U(t), t)=|U(t)| \log |U(t)|+h(t) .
\end{gathered}
$$

Conditions (a)-(c) assure that if (2.5)-(2.6) is identified with (2.20), then the assumptions of Propositions 2.3 are fulfilled. Condition (2.23) implies that $u=v=w=x=0$ is the solution of (2.20) satisfying the initial condition $u(0)=v(0)$ $=w(0)=x(0)=0$, while (2.24) implies that $u=U(t), v=U^{\prime}(t), w=t, x=U(t)+1$ is the solution satisfying $u(0)=v(0)=w(0)=(0), x(0)=1$. Since $U(t)$ is not positive for small $t>0$, it follows that $(v, w, x)$ are not pseudoderivatives of $u$. Hence the proof is complete if the function $q$ is exhibited. 
There exists a continuous increasing function $m(t)$ on $t \geqq 0$ satisfying $m(0)=0$ and

$$
h(t) / m(t) \rightarrow 0 \text { as } t \rightarrow+0 .
$$

Define a function $g(t, w)$ as follows:

$$
\begin{aligned}
& g(t, w)=w \text { for } t \leqq 0 \text { and for } 0<t<b, w<0, \\
& g(t, w)=m(w) h(t) / m(t) \text { for } 0 \leqq w \leqq 2 t(<2 b), \\
& g(t, w)=m(2 t) h(t) / m(t)+w-2 t \text { for } 0<2 t \leqq w<\infty .
\end{aligned}
$$

Let $f(u)$ be defined by

$$
f(u)=|u| \log |u| \text { or } f(u)=0,
$$

according as $0<|u| \leqq 1$ or $u=0$ and $|u|>1$. Finally,

$$
q(t, u, w)=f(u)+g(t, w) .
$$

Condition (a) on $q$ is obvious; (b) follows from Osgood's uniqueness criterion since, for small $\left|u_{1}\right|+\left|u_{2}\right|$,

$$
\left|f\left(u_{1}\right)-f\left(u_{2}\right)\right| \leqq-\left|u_{1}-u_{2}\right| \log \left|u_{1}-u_{2}\right| \text { and } \int_{+0}(-u \log u)^{-1} d u=\infty \text {; }
$$

(c) follows from (2.27); and (2.23), (2.24) are clear from (2.27) and from (2.28). Thus, in order to complete the proof, it suffices to verify the lemma.

Proof of the lemma. Define the sequences

$$
\begin{aligned}
& a_{n}=2^{-n} \text { and } b_{n}=2^{-n-1} \text { for } n=0,1, \ldots, \\
& c_{n}=c_{n+1}=2^{2 n+4} \exp \left(-2^{2 n+5}\right) \text { for odd } n .
\end{aligned}
$$

Note that $b_{n}=2 b_{n+1}$,

$$
c_{n+1} / c_{n} \rightarrow 0 \text { as even } n \rightarrow \infty
$$

and that

$$
b_{n} c_{n} \geqq 2 b_{n+1} c_{n+1} \text { for all } n .
$$

On $[0,1]$, define the function $U(t)$ by quadratures, $(2.19)$ and

$$
\begin{aligned}
& U^{\prime \prime}(t)=(-1)^{n}\left(\pi c_{n} / 2\right) \sin \pi\left(t-a_{n}\right) / b_{n} \quad \text { if } a_{n} \leqq t \leqq a_{n}+b_{n}, \\
& U^{\prime \prime}(t)=0 \quad \text { otherwise. }
\end{aligned}
$$

On the interval $\left[a_{n}+b_{n}, a_{n-1}\right], U^{\prime}(t)$ is the constant

$$
U^{\prime}\left(a_{n}+b_{n}\right)=U^{\prime}\left(a_{n-1}\right)=(-1)^{n} s_{n},
$$

where

$$
s_{n}=(-1)^{n} \sum_{m=n}^{\infty}(-1)^{m} b_{m} c_{m} .
$$


Thus $U(t)$ is linear and, by (2.29),

$$
U\left(a_{n-1}\right)=U\left(a_{n}+b_{n}\right)+(-1)^{n} b_{n} s_{n} .
$$

On the interval $\left[a_{n}, a_{n}+b_{n}\right], U^{\prime}(t)$ is monotone and, in fact,

$$
U^{\prime}(t)=U^{\prime}\left(a_{n}\right)+(-1)^{n} \frac{1}{2} b_{n} c_{n}\left[1-\cos \pi\left(t-a_{n}\right) / b_{n}\right] .
$$

From (2.34) and (2.37),

$$
U\left(a_{n}+b_{n}\right)=U\left(a_{n}\right)+(-1)^{n}\left[\frac{1}{2} b_{n}^{2} c_{n}-b_{n} s_{n+1}\right] .
$$

Consequently, (2.36) and (2.38) give the recursion formula $U\left(a_{n-1}\right)=U\left(a_{n}\right)+$ $(-1)^{n}\left[\frac{1}{2} b_{n}^{2} c_{n}+b_{n} s_{n}-b_{n} s_{n+1}\right]$, so that

$$
U\left(a_{n}\right)=\sum_{m=n+1}^{\infty}(-1)^{m}\left(\frac{1}{2} b_{m}^{2} c_{m}+b_{m} s_{m}\right)-\sum_{m=n+1}^{\infty}(-1)^{m} b_{m} s_{m+1} .
$$

The series in (2.35) is an alternating series with monotone terms and, by (2.32), the same holds for the series for $s_{n}-s_{n+1}$, so that

$$
s_{n} \geqq s_{n+1}>0 .
$$

We have $b_{n} c_{n}-b_{n+1} c_{n+1} \leqq s_{n} \leqq b_{n} c_{n}$, hence

$$
\frac{1}{2} b_{n} c_{n} \leqq s_{n} \leqq b_{n} c_{n} \text {. }
$$

Furthermore, by (2.31),

$$
\begin{array}{ll}
s_{n} \sim b_{n} c_{n} & \text { as even } n \rightarrow \infty, \\
s_{n} \sim \frac{1}{2} b_{n} c_{n} & \text { as odd } n \rightarrow \infty
\end{array}
$$

Note that (2.34) gives

$$
U^{\prime}\left(a_{n-1}\right) U^{\prime}\left(a_{n}\right)<0 .
$$

We shall verify that

$$
U\left(a_{n-1}\right) U\left(a_{n}\right)<0 \text { for large } n .
$$

In fact, (2.31) and (2.39)-(2.43) imply that

$$
U\left(a_{n}\right) \sim \frac{1}{2} b_{n+1}^{2} c_{n+1}+b_{n+1}^{2} c_{n+1}=(3 / 8) b_{n}^{2} c_{n} \text { for odd } n,
$$

while for even $n$,

$$
-U\left(a_{n}\right) \sim\left[\left(\frac{1}{2} b_{n+1}^{2} c_{n+1}+b_{n+1} s_{n+1}\right)-\left(\frac{1}{2} b_{n+2}^{2} c_{n+2}+b_{n+2} s_{n+2}\right)\right]-b_{n+1} s_{n+2},
$$

so that

$$
-U\left(a_{n}\right) \sim(1 / 8) b_{n+1}^{2} c_{n+1}>0 \text { as even } n \rightarrow \infty .
$$

By (2.42), as odd $n \rightarrow \infty$, the quantity [...] in (2.38) satisfies

$$
\frac{1}{2} b_{n}^{2} c_{n}-b_{n} s_{n+1}=\frac{1}{2} b_{n}^{2} c_{n}-(1+o(1)) b_{n} b_{n+1} c_{n+1}=o(1) b_{n}^{2} c_{n} .
$$


Thus, by (2.42) and (2.46),

$$
U\left(a_{n}\right) \sim U\left(a_{n}+b_{n}\right) \sim(3 / 8) b_{n}^{2} c_{n}>0 \text { as odd } n \rightarrow \infty .
$$

On the other hand, for even $n$,

$$
U\left(a_{n}+b_{n}\right)-U\left(a_{n}\right)=\frac{1}{2} b_{n}^{2} c_{n}-b_{n} s_{n+1} \sim \frac{1}{2} b_{n}^{2} c_{n} ;
$$

so that, by (2.47),

$$
U\left(a_{n}+b_{n}\right)>0>U\left(a_{n}\right) \text { for large even } n .
$$

Since $U^{\prime \prime}(t) \leqq 0$ on $\left[a_{n}, a_{n}+b_{n}\right]$ for odd $n,(2.46)$ and (2.48) show that

$$
U(t) \geqq \min \left[U\left(a_{n}\right), U\left(a_{n}+b_{n}\right)\right] \sim(3 / 8) b_{n}^{2} c_{n} \text { for large odd } n .
$$

Thus, for odd $n, U(t)>0$ on $\left[a_{n}, a_{n}+b_{n}\right]$ and then changes sign once in $\left[a_{n}+b_{n}, a_{n-1}\right]$. While, for even $n, U(t)$ changes sign once on $\left[a_{n}, a_{n}+b_{n}\right]$, by (2.49) and $U^{\prime \prime} \geqq 0$, and $U(t)>0$ on $\left[a_{n}+b_{n}, a_{n-1}\right]$.

Choose $b, 0<b<1$, so small that $|U|<\delta$ on $[0, b]$, where $-u \log u$ is increasing on $0<u \leqq \delta<1$, and that the inequalities above, specified to hold for large $n$, are valid if $a_{n-1}<b$. On $[0, b]$, define $h(t)$ by (2.18). It is clear that $h(t)$ is continuous. It has to be verified that $h(t)>0$ on $(0, b]$. Since $h(t)>0$ whenever $0<t \leqq b$ and $U^{\prime \prime}(t) \geqq 0$, it suffices to examine $h(t)$ for $t$ on $\left[a_{n}, a_{n}+b_{n}\right]$ for $n$ odd, where $0 \leqq-U^{\prime \prime}(t) \leqq \pi c_{n} / 2 \leqq 2 c_{n}$. In view of $(2.50), h(t)>0$ holds on $\left[a_{n}, a_{n}+b_{n}\right]$, provided that $2 c_{n} \leqq-\left(b_{n}^{2} c_{n} / 4\right) \log \left(b_{n}^{2} c_{n} / 4\right)$. Since this inequality is clear from (2.29) and (2.30), the proof is complete.

3. On different sets of pseudoderivatives. This section is concerned with the following:

Propgsition 3.1. Let $\mathscr{F}=\left\{\left(f^{0} ; f^{1}, \ldots, f^{N-1}\right)\right\} \quad$ and $\mathscr{F}_{*}=\left\{\left(f^{0} ; f_{*}^{1}, \ldots, f_{*}^{N-1}\right)\right\}$ denote families $F$ of the same continuous functions $f^{0}$ on an open interval $J^{0}$ with pseudoderivatives, $\left(f^{1}, \ldots, f^{N-1}\right)$ and $\left(f_{*}^{1}, \ldots, f_{*}^{N-1}\right)$, having the property of unique initial values. Let $t_{0} \in J^{0}$ be fixed. Then there exists a one-to-one map of $R^{N}$ onto $R^{N}$, say, $z=g(y)$ or

$$
z_{i}=g_{i}\left(y_{0}, \ldots, y_{N-1}\right) \text { for } i=0, \ldots, N-1
$$

such that $g_{0}\left(y_{0}, \ldots, y_{N-1}\right) \equiv y_{0}$ and

$$
f_{*}^{i}\left(t_{0}\right)=g_{i}\left(f^{0}\left(t_{0}\right), f^{1}\left(t_{0}\right), \ldots, f^{N-1}\left(t_{0}\right)\right) \text { for } i=1, \ldots, N-1 .
$$

If, in addition, the one-to-one maps $R^{N} \leftrightarrow R^{N}$, given by $y \leftrightarrow z=g(y)$, are continuous, then

$$
g_{i}=g_{i}\left(y_{0}, \ldots, y_{i}\right) \text { for } i=0, \ldots, N-1
$$

is a function of $\left(y_{0}, \ldots, y_{i}\right)$, independent of $\left(y_{i+1}, \ldots, y_{N-1}\right)$, and is an increasing function of $y_{i}$ satisfying

$$
g_{i}\left(y_{0}, \ldots, y_{i}\right) \rightarrow \pm \infty \text { as } y_{i} \rightarrow \pm \infty \text {. }
$$


It remains undecided whether or not the last part of this proposition is correct if it is assumed that (3.1) is a homeomorphism $R^{N} \rightarrow R^{N}$.

Proof. The existence of a one-to-one map $R^{N} \leftrightarrow R^{N}$, given by (3.1), and satisfying $g_{0} \equiv y_{0}$ and (3.2) is clear from the fact that the families $\mathscr{F}_{,} \mathscr{F}_{*}$ have the property of unique initial values. Assume that the map (3.1) and its inverse are continuous.

We first show that $g_{1}\left(y_{0}, \ldots, y_{N-1}\right)$ is independent of $\left(y_{2}, \ldots, y_{N-1}\right)$. It is clear that $g_{1}$ is a nondecreasing function of $y_{j}, j>0$; cf. (3.2) and Remark 2 in $\$ 1$. Suppose that $g_{1}$ is not independent of $y_{2}$. Then there exist $y_{2}$-values, say $y_{2}=u, v$, and $\left(t, y_{0}, y_{1}\right),\left(y_{3}, \ldots, y_{N-1}\right)$ such that $u<v$ and

$$
g_{1}\left(y_{0}, y_{1}, u, y_{3}, \ldots, y_{N-1}\right)<g_{1}\left(y_{0}, y_{1}, v, y_{3}, \ldots, y_{N-1}\right) .
$$

By continuity, the same inequality holds if $y_{1}$ on the right is replaced by $y_{1}-\varepsilon$ for small $\varepsilon>0$. But (3.2) and Remark 2 in $\S 1$ imply that

$$
g_{1}\left(y_{0}, y_{1}, u_{2}, u_{3}, \ldots, u_{N-1}\right) \geqq g_{1}\left(y_{0}, y_{1}-\varepsilon, v_{2}, v_{3}, \ldots, v_{N-1}\right)
$$

for all $\left(t_{0}, y_{0}, y_{1}\right),\left(u_{2}, \ldots, u_{N-1}\right),\left(v_{2}, \ldots, v_{N-1}\right)$ and $\varepsilon>0$. This contradiction shows that $g_{1}$ is independent of $y_{2}$. Similarly, it is seen to be independent of $y_{3}, \ldots, y_{N-1}$.

We now show that $g_{2}\left(y_{0}, \ldots, y_{N-1}\right)$ is independent of $\left(y_{3}, \ldots, y_{N-1}\right)$. To this end, let $t_{0}$ and $y_{1}$ be fixed (but $y_{0}, y_{2}, \ldots, y_{N-1}$ arbitrary) and consider the subfamily $F_{0}$ of $F$ consisting of elements $f^{0}$ in $F$ satisfying the initial condition $f^{1}\left(t_{0}\right)=y_{1}$. Then the family $\mathscr{F}_{1}=\left\{\left(f^{0}, f^{2}, \ldots, f^{N-1}\right)\right\}$ has the property that $f^{0} \in F_{0}$ has the "pseudoderivatives" $\left(f^{2}, \ldots, f^{N-1}\right)$ at $t=t_{0}$ if one only examines $f^{0}$ for $t \geqq t_{0}$. The corresponding subfamily $F_{* 0}$ of $F_{*}$ is determined by $f^{0}\left(t_{0}\right)=y_{0}, f_{*}^{1}\left(t_{0}\right)=g_{1}\left(y_{0}, y_{1}\right)$, when $t_{0}, y_{1}$ are fixed, and $f^{0} \in F_{*}$ has the " pseudoderivatives" $f_{*}^{2}, \ldots, f_{*}^{N-1}$ at $t=t_{0}$ if one only considers $t \geqq t_{0}$.

The argument above, showing that $g_{1}$ is independent of $y_{2}, \ldots, y_{N-1}$, can be used to show that $g_{2}\left(y_{0}, y_{1}, \ldots, y_{N-1}\right)$, for fixed $y_{1}$, is independent of $y_{3}, \ldots, y_{N-1}$. This induction continues and we obtain (3.3).

Since the map of $R^{N}$ onto $R^{N}$ which is the inverse of (3.1) has a form similar to (3.3), it follows that $g_{i}$ is an increasing function of $y_{i}$ and that (3.4) holds. Hence the proof is complete.

\section{REFERENCES}

1. O. Aramă, Rezultate comparative asupra unor probleme la limita pentru ecuatii differentiale lineare, Acad. R. P. Romîne. Fil. Cluj. Stud. Circ. Mat. 10 (1959), 207-257.

2. E. F. Beckenbach, Generalized convex functions, Bull. Amer. Math. Soc. 43 (1937), 363-371.

3. R. Conti, Recent trends in the theory of boundary value problems for ordinary differential equations, Boll. Un. Mat. Ital. (3) 22 (1967), 135-178. MR 36 \#1734.

4. M. Fukuhara, Sur l'ensemble des courbes intégrales d'un système d'équations différentielles ordinaires, Proc. Imp. Acad. Japan 6 (1930), 360-362.

5. P. Hartman, Unrestricted n-parameter families, Rend. Circ. Mat. Palermo (2) 7 (1958), 123-142. MR 21 \#4211.

6. ——, Ordinary differential equations, Wiley, New York, 1964. MR 30 \#1270. 
7. E. Kamke, Zur Theorie der Systeme gewöhnlicher Differentialgleichungen. II, Acta Math. 58 (1932), 57-85.

8. A. Lasota and M. Luczyński, A note on the uniqueness of two point boundary value problems. I, Zeszyty Nauk. Uniw. Jagiello, Prace Mat. No. 12 (1968), 27-29. MR 37 \#499.

9. A. Lasota and Z. Opial, L'existence et l'unicité des solutions du problème d'interpolation pour l'équation différentielle ordinaire d'ordre n, Ann. Polon. Math. 15 (1964), 253-271. MR 30 \#4012.

10. - On the existence and uniqueness of solutions of a boundary value problem for an ordinary second-order differential equation, Colloq. Math. 18 (1967), 1-5. MR 36 \#2871.

11. L. S. Nicolson, Boundary value problems for systems of ordinary differential equations, J. Differential Equations 6 (1969), 397-407.

12. Z. Opial, On a theorem of $O$. Aramă, J. Differential Equations 3 (1967), 88-91. MR 34 \#6194.

13. M. M. Peixoto, Generalized convex functions and second order differential inequalities, Bull. Amer. Math. Soc. 55 (1949), 563-572. MR 10, 686.

14. M. C. Peixoto, On the inequalities $y^{\prime \prime} \geqq G\left(x, y, y^{\prime}, y^{\prime \prime}\right)$, An. Acad. Brasil Ci. 21 (1949), 205-218.

15. L. Tornheim, On n-parameter families of functions and associated convex functions, Trans. Amer. Math. Soc. 69 (1950), 457-467. MR 12, 395.

16. W. M. Whyburn, Differential systems with boundary conditions at more than two points, Proc. Conference Differential Equations, Univ. of Maryland Book Store, College Park, Md., 1956. MR 18, 481.

17. K. W. Schrader and P. Waltman, An existence theorem for non-linear boundary value problems, Proc. Amer. Math. Soc. 21 (1969), 653-656. MR 39 \#533.

THE JOHNS HOPKINS UNIVERSITY,

Baltimore, MARYLAND 21218

Courant Institute of Mathematical Sciences, New York University, NEW YORK, NeW YORK 10012 